\title{
Investigation of Twin Jet Aeroacoustic Properties in the Presence of a Hybrid Wing Body Shield
}

\author{
Michael J. Doty ${ }^{1}$ \\ NASA Langley Research Center, Hampton, VA, 23681
}

\begin{abstract}
In preparation for upcoming wind tunnel acoustic experiments of a Hybrid Wing Body (HWB) vehicle with two jet engine simulator units, a series of twin jet aeroacoustic investigations were conducted leading to increased understanding and risk mitigation. A previously existing twin jet nozzle system and a fabricated HWB aft deck fuselage are combined for a $1.9 \%$ model scale study of jet nozzle spacing and jet cant angle effects, elevon deflection into the jet plume, and acoustic shielding by the fuselage body. Linear and phased array microphone measurements are made, and data processing includes the use of DAMAS (Deconvolution Approach for the Mapping of Acoustic Sources). Closely-spaced twin jets with a $5^{\circ}$ inward cant angle exhibit reduced noise levels compared to their parallel flow counterparts at similar and larger nozzle spacings. A $40^{\circ}$ elevon deflection into the twin jet plume, which is required for HWB ground rotation, can significantly increase upstream noise levels (more than $5 \mathrm{~dB}$ OASPL) with only minimal increases in the downstream direction. Lastly, DAMAS processing can successfully measure the noise source distribution of multiple shielded jet sources.
\end{abstract}

\section{Nomenclature}

$a_{m}$

Greek

$\gamma_{i j}^{2} \quad=$ Coherence function

$\varphi_{i j} \quad=$ Phase angle

$\theta \quad=$ Angle from jet inlet axis

\footnotetext{
${ }^{1}$ Research Aerospace Engineer, Aeroacoustics Branch, Mail Stop 461, Senior Member AIAA.
} 


$\begin{array}{ll}\tau_{m} & =\text { Propagation time from grid point } n \text { to microphone } m \\ \Delta \tau_{i j} & =\text { Time delay between microphone pairs } \\ \text { Subscript } & \\ j & =\text { jet exit quantity } \\ m & =\text { microphone number } \\ n & =\text { scanning grid point number } \\ W T & =\text { wind tunnel quantity }\end{array}$

\section{Introduction}

A ircraft with more than one jet engine are common in aviation today. In many military, business jet, and commercial aircraft applications these jet engine exhausts are often spaced closely to one another. In particular, two jet nozzles of identical geometry operating at the same flow conditions, i.e. twin jets, are often found in rear-mount or aft-tail mount configurations, or under one wing of a four-engine aircraft.

Because jet noise is a dominant noise source for many aircraft, the effects twin jets can have on the noisegenerating flowfields and the propagation paths of one another have been a topic of study for several decades. Greatrex and Brown ${ }^{1}$ showed a noise reduction using twin nozzles in 1958. Several investigations of twin jet acoustics occurred in the late 1970's and early 1980's in the context of multi-lobe jet noise suppressor technologies, such as Borchers ${ }^{2}$, Bhat ${ }^{3}$, Parthasarathy, et al. $^{4}$ and Kantola ${ }^{5}$, to name a few. Further work included a coordinated effort between $\mathrm{Yu}$ and Fratello ${ }^{6}$ and Gerhold ${ }^{7}$ to measure and model the shielding of a point source by a jet flow, thereby isolating the acoustic jet-jet shielding effect from the jet flowfield interaction effect. The jet-jet shielding effect is actually a result of several mechanisms, as discussed by Simonich, et al. ${ }^{8}$, including: reflection (in which sound waves from the source jet are cast back due to the impedance mismatch between the shielding jet and surrounding air), refraction (in which the sound waves change direction as they pass through the shielding jet), diffraction (in which the sound waves travel around the shielding jet barrier), and scattering (in which the sound waves are dispersed by the turbulent flow of the shielding jet). The detailed parametric study by Simonich, et al. ${ }^{8}$ showed that shielding up to $3 \mathrm{~dB}$ was possible in the plane of two jets but must be weighed in the context of the equally significant increases in noise occurring in other azimuthal directions due to the reflection, refraction, and diffraction effects of the adjacent jet flowfield. Furthermore, at close spacings in which the two jet plumes merge and alter the acoustic sources, low frequency noise increases were observed ${ }^{8}$.

A more recent investigation of twin jet shielding at subsonic and supersonic conditions was undertaken by Bozak and Henderson'. A $3 \mathrm{~dB}$ overall sound pressure level (OASPL) noise reduction was also observed in this study in the plane of the two jets in the peak noise direction. Conversely, a $3 \mathrm{~dB}$ OASPL noise increase was measured in the out-of-plane azimuthal direction. As these authors point out, future vehicle concepts may utilize acoustic benefits from jet-jet shielding. If the azimuthal directions of observed twin jet noise increases (namely, the out-of-plane direction) could be shielded by the fuselage body, further benefits could be realized.

The current experiments investigate two closely-spaced jets with and without the presence of a wing shield. The intention is to maximize engine noise reduction using not only jet-jet shielding but also fuselage shielding by placing the engines over a Hybrid Wing Body (HWB) planform in preparation for a larger future test. That future test is the upcoming aeroacoustics test of a 5.8\% HWB scale model which will take place in the NASA Langley 14 by 22 foot wind tunnel. Figure 1 shows the HWB model during recent aerodynamic testing. The aeroacoustics test will include two Compact Jet Engine Simulators (CJES) mounted underneath the inverted model as shown in Fig. 2. While the jets will benefit from jet-jet shielding in some azimuthal directions, the extended trailing edge of the HWB will also serve to shield the propagating jet noise. However, the stability and control characteristics of this flying wing design require a constant upward elevon deflection of $10^{\circ}$ during normal operations. An increased upward elevon deflection of $40^{\circ}$ is required during rotation off the runway. Unlike the $10^{\circ}$ deflection, the $40^{\circ}$ deflection actually impinges into the jet plumes, and the acoustic impacts due to the jet flows scrubbing the deflected elevon surface have not been characterized. 


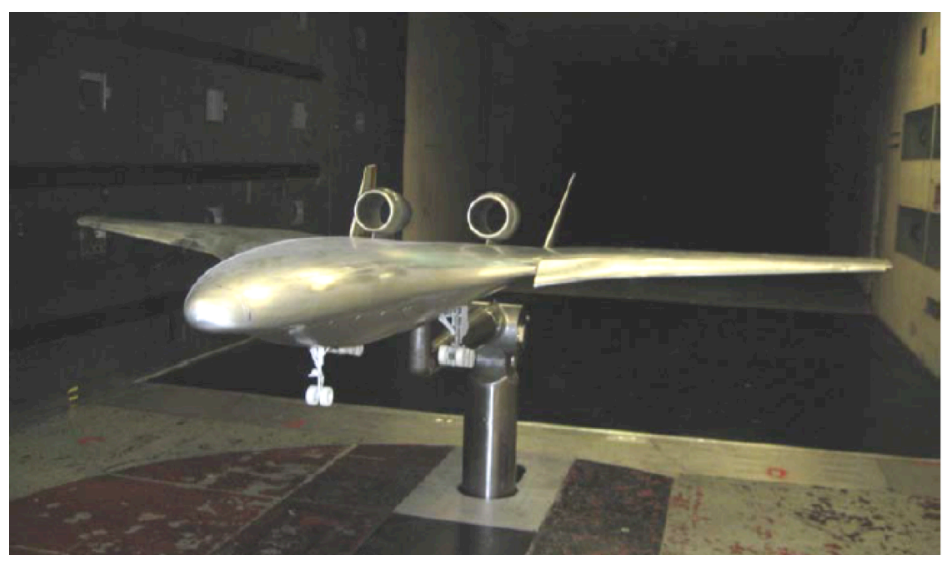

Figure 1. 5.8\% HWB model in the $14 \times 22 \mathrm{ft}$ wind tunnel during the aerodynamics test.

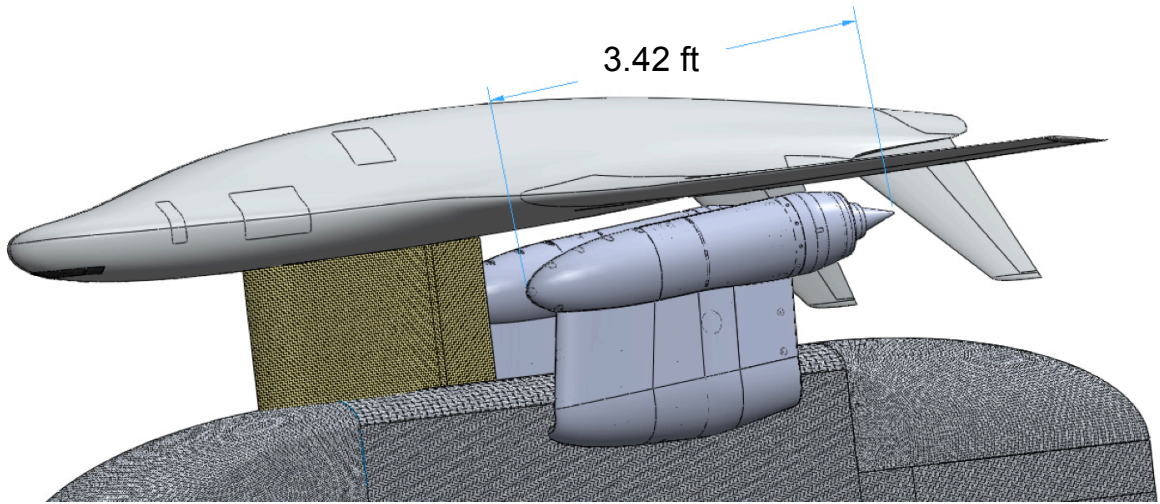

Figure 2. Schematic of the CJES mounted under the inverted HWB model for the aeroacoustics test.

Furthermore, while these jets' exhausts are not canted inward, future designs could benefit from such an arrangement because it would, in turn, allow the engine inlets to be canted outward, more precisely aligning with the flow over the wing body and reducing inlet distortion. The acoustic effects of canted twin jets for this application have not been explored. Lastly, the predominant measurement technique for the wind tunnel test will be a 97microphone phased array used in conjunction with the DAMAS array processing technique described by Brooks and Humphreys ${ }^{10}$. The measurement of two distributed jet noise sources in the presence of shielding and partial shielding environments represents a significant challenge for the DAMAS technique.

The current study serves to investigate the aforementioned issues to mitigate risks and increase understanding prior to the $14 \times 22$ entry. An existing twin jet model system (albeit single stream for each nozzle) is used in conjunction with an HWB aft-deck fuselage model that is approximately $1.9 \%$ of full scale as shown in Fig. 3 . The $1.9 \%$ scale assumes each twin jet nozzle diameter represents a fan stream diameter of the CJES unit. Each singlestream twin jet nozzle simulates the combined fan and core streams of a CJES unit by utilizing the mass-weighted mixed conditions of the fan and core streams at representative HWB takeoff conditions. Mean flowfield, acoustic, and phased array measurements are made of this system to investigate the aeroacoustic effects of the canted jets, the acoustic impact of the upward elevon deflection, and the phased array source location effectiveness for shielded twin jets. Section II of this paper describes the facility, hardware, instrumentation, and data processing in greater detail. Section III discusses mean flowfield and acoustic measurements, including both single microphone and phased array results. Lastly, Section IV presents a summary of the results and draws relevant conclusions. 


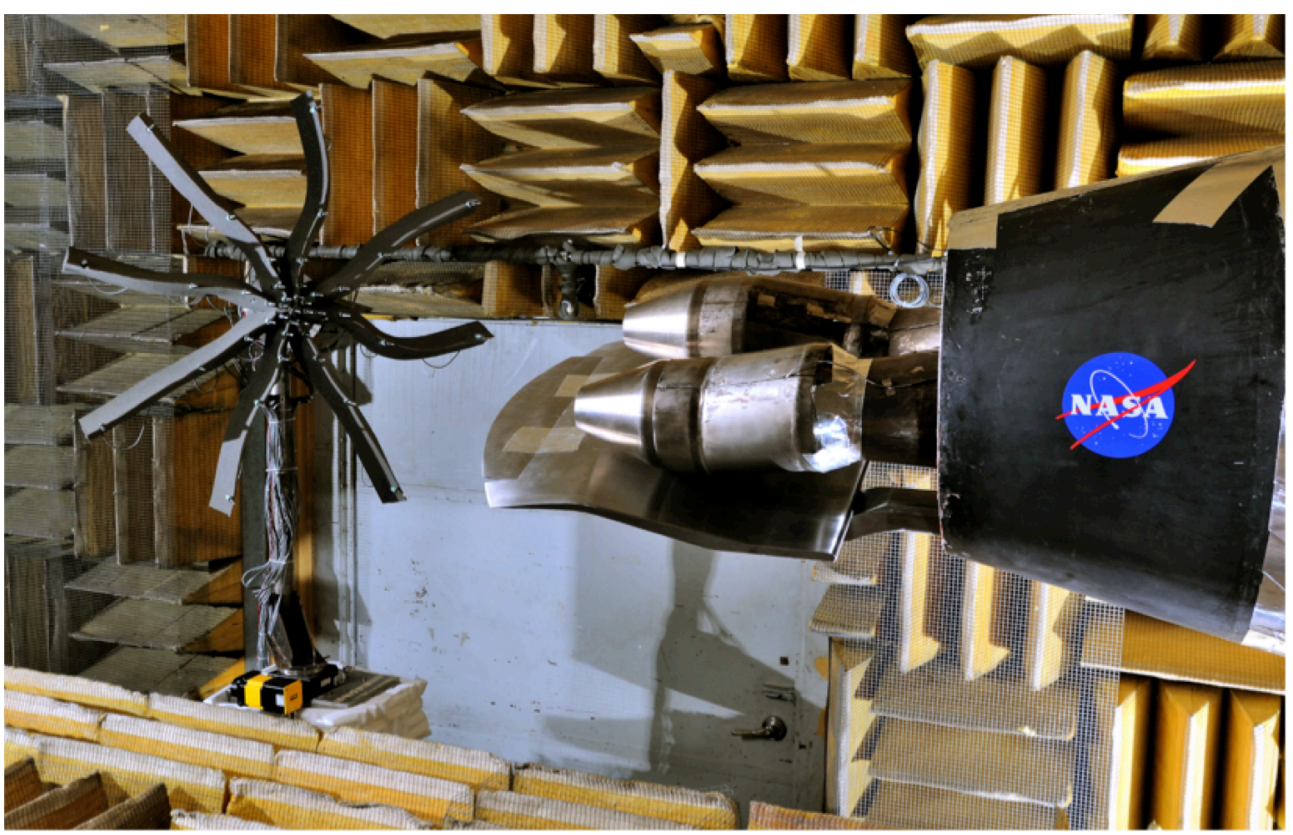

Figure 3. HWB aft deck fuselage model installed below the twin jet with the phased array in the background. (Wedges are placed in front of the test cell door during acoustic testing.)

\section{Experimental Procedures}

\section{A. Facility and Nozzle System Description}

The twin jet experiments took place in the NASA Langley Research Center Jet Noise Lab (JNL) within the Low Speed Aeroacoustic Wind Tunnel (LSAWT) shown in Fig. 4. The LSAWT is comprised of a $17.5 \mathrm{ft}$ high x $17 \mathrm{ft}$ wide x $34 \mathrm{ft}$ long anechoic chamber surrounding an open jet wind tunnel and a dual-stream jet engine simulator (JES). The wind tunnel simulates forward flight using a $4.7 \mathrm{ft}$ x $4.7 \mathrm{ft}$ free jet capable of speeds up to Mach 0.32 . The JES is centered within this free jet and is capable of delivering up to $20 \mathrm{lbm} / \mathrm{sec}$ of air per stream and nozzle pressure ratios up to 12. The JES provides independent control of both core and bypass streams at temperatures up to $2000^{\circ} \mathrm{F}$ through the use of sudden expansion liquid propane burners and water cooling.

The twin jet model is mounted on the JES core duct as shown in Fig. 5a. The model is also described in Bozak and Henderson ${ }^{9}$ and consists of a y-duct splitting off from a common plenum, two s-ducts which, when rotated, permit changes in spacing between nozzles, two straight (or angle) adapters, and two nozzles. The straight adapters are used when no cant angle is desired, and the $5^{\circ}$ angle adapters are used to produce a $5^{\circ}$ inward cant angle for each nozzle. In the current work, two-inch diameter convergent conical nozzles are used at various centerline-tocenterline spacings (S). The azimuthal orientation of the twin jet model with respect to the microphones is also variable. The results of the current work are predominantly with the twin jet model either "in-plane" or "out-ofplane" of the linear array or phased array microphones. As depicted in Fig. 5b, the "in-plane" configuration refers to an orientation in which the plane containing the linear microphone array or the horizontal plane containing the JEDA center microphone also intersects both nozzle centerlines. The "out-of-plane" configuration refers to an orientation of the microphone plane that is perpendicular to the axis connecting the two nozzle centerlines. 


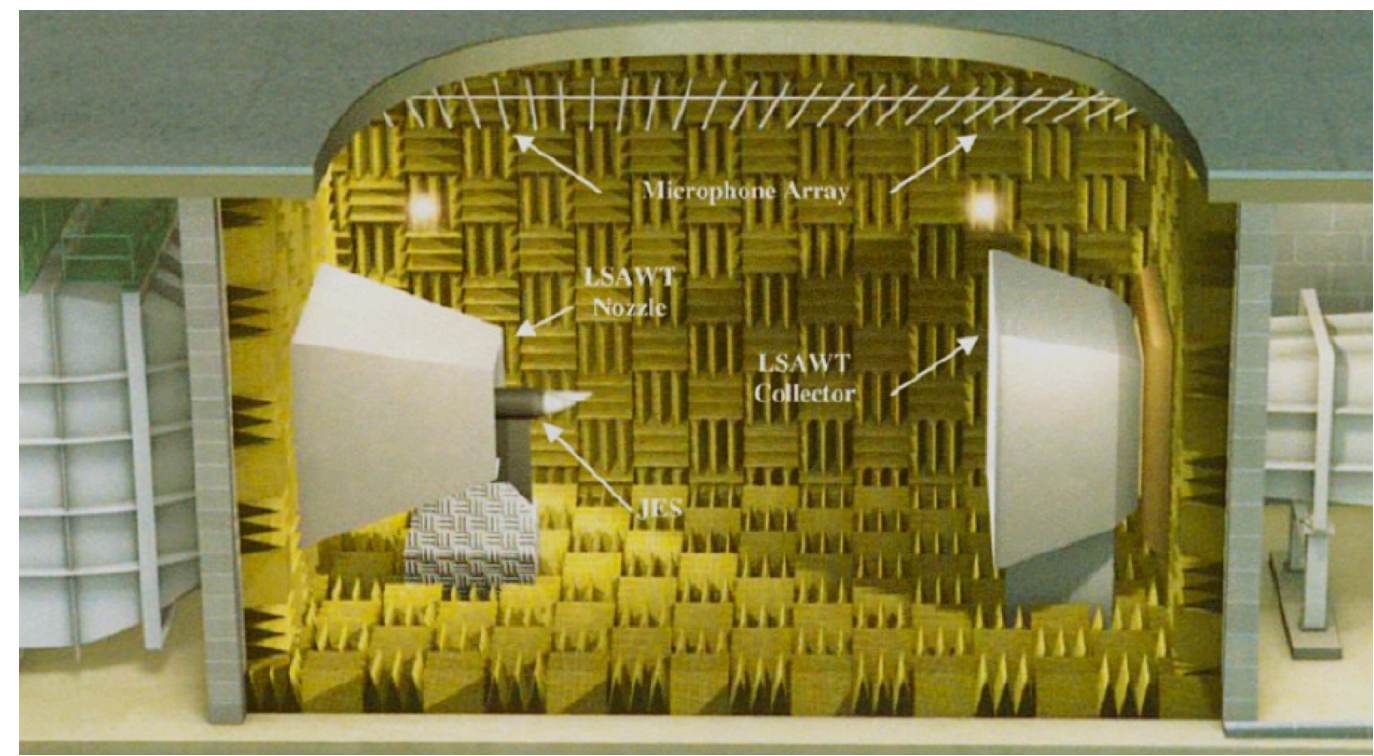

Figure 4. NASA Langley Low Speed Aeroacoustic Wind Tunnel.

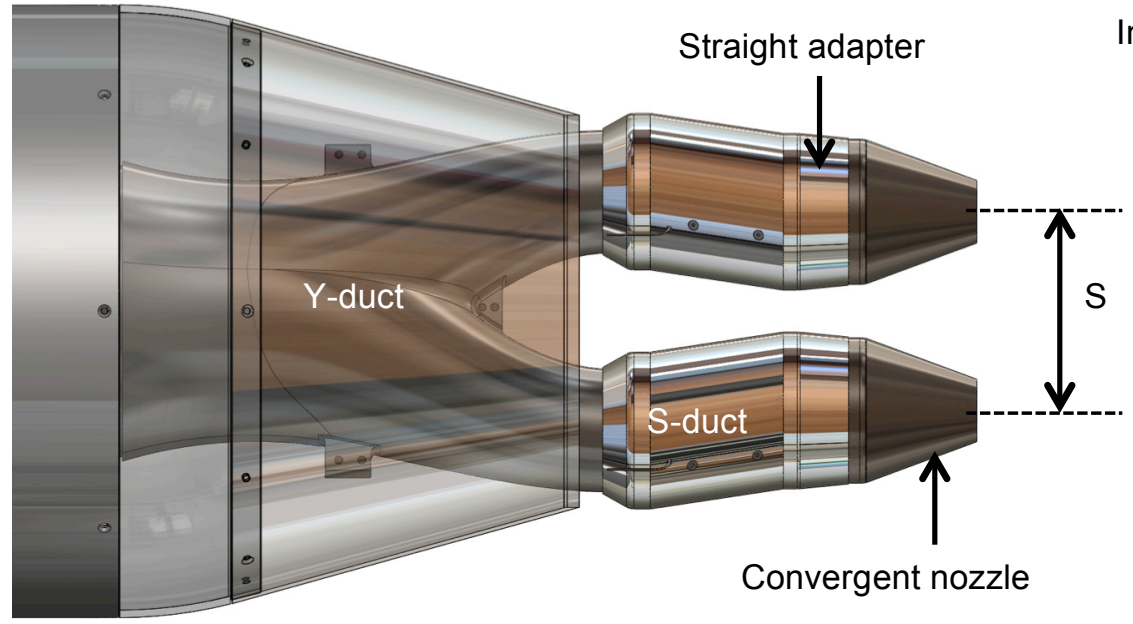

In-plane

a)

b)

Figure 5. Twin jet nozzle system a) installed on the Jet Engine Simulator (JES) and b) with azimuthal orientations shown.

\section{B. Instrumentation}

1. Pressure Transducers and Thermocouples for Flowfield Measurements

A nine-probe survey rake of alternating total pressure and total temperature probes spaced in one-inch increments was mounted to a three-axis traverse system within the facility. The pressure ports were connected to a Pressure Systems Series 8400 ESP Pressure Scanner ( \pm 45 psid), and the temperature probes were Type K thermocouples with Inconel sheaths (0.062" diameter). The survey rake provided simultaneous measurement of 5 total pressures and 4 total temperatures at each measurement station. Typical grid point spacing (i.e. resolution) for a plume map was $0.25-0.50$ inches, depending on axial location. Therefore, the generation of a plume map normally required 300-500 probe movements, which were automated as part of the LabVIEW data acquisition program. 


\section{Phased Array}

The JEt noise Directional Array (JEDA) consists of 41 Brüel and Kjær (B\&K) Model 4939 free-field microphones of 0.25 inch diameter. Model 2670 and Model 2669 pre-amplifiers and B\&K 5935L 2-channel power supplies were connected to the microphones. The microphones were mounted within phenolic sleeves on an aluminum frame which was covered with acoustic foam as shown in Fig. 3. The array pattern design is described in Brooks, et al. ${ }^{11}$ and includes a center microphone and 5 concentric rings of 8 microphones each, with various azimuthal spacing of the microphones for each ring. The largest ring is 48 inches in diameter with each subsequent inner ring being half the diameter of the previous one. The JEDA was mounted on a foam covered traverse table with the array face located 72 inches from the centerline between jets. For the current work the JEDA was positioned $90^{\circ}$ from the upstream jet axis and also traversed downstream 72 inches and rotated toward the jet nozzle, resulting in a location of $135^{\circ}$ from the upstream jet axis as shown in Fig. 6. Single microphone spectra are shown at both $90^{\circ}$ and $135^{\circ}$, but the processed phased array results in this work will focus on the $90^{\circ}$ location. Three additional wall microphones were placed just behind the JEDA at $90^{\circ}, 117^{\circ}$, and $135^{\circ}$ for reference. Injection and pistonphone calibrations were performed routinely on all microphones, and the microphone grid caps were removed during testing. In addition, the pointing accuracy of the array was checked using two broadband monopole point sources. These calibration point sources were Selenium DH200 acoustic drivers connected to a 0.625 inch pipe open at the opposite end. A waveform generator and power amplifier were used to excite the driver with a white noise signal. The calibration point sources were used to correct for unexpected phase differences between array microphones due to propagation through the wind tunnel shear layer and/or imprecise array positioning.

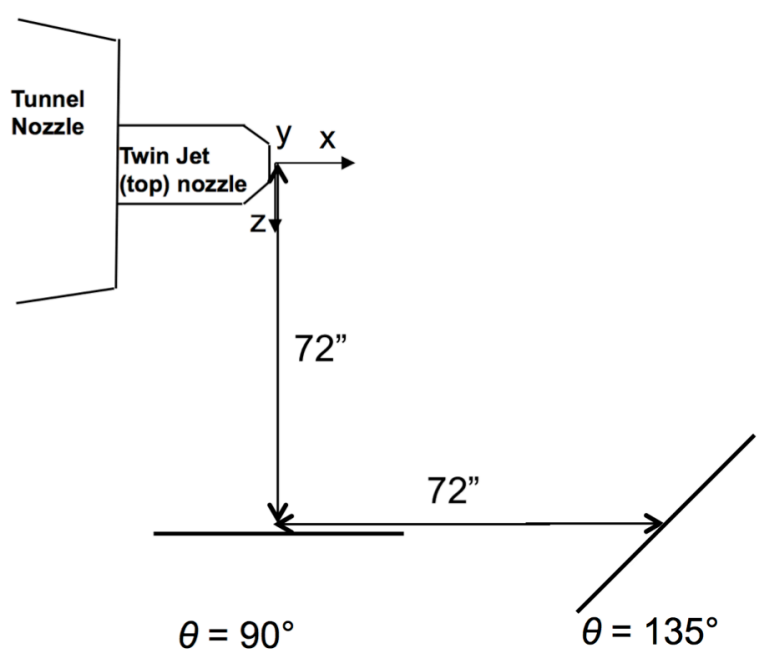

Figure 6. Overhead schematic of JEDA positioning with respect to twin jet model (out-of-plane).

\section{Far-Field Microphones}

Although not the primary acoustic instrumentation for this test, the standard LSAWT linear array of $28 \mathrm{~B} \& \mathrm{~K}$ Model 4939 free-field microphones of 0.25 inch diameter was, nonetheless, used to measure far-field noise characteristics in conjunction with Model 2670 pre-amplifiers and 8 B\&K Type 2829 4-channel power supplies. The angular range of the microphone positions was from $\theta=40^{\circ}$ to $150^{\circ}$ from the upstream jet axis. The microphones were located on an azimuthal angle of $50^{\circ}$ above the sideline and 138.5 inches away from the centerline between jets, pointed toward the twin jet exit plane. Electrostatic and pistonphone calibrations were routinely performed on all microphones, and the grid caps were removed for testing. 


\section{Data Acquisition and Processing}

\section{Single Sensor}

The standard LSAWT data acquisition system (DAS) consists of a series of LabVIEW virtual instruments running on an acquisition PC. The steady-state pressure transducer and thermocouple signals were averaged over 5 seconds while the test point was maintained within $0.5 \%$ for nozzle pressure ratio (NPR) and within $1 \%$ for nozzle temperature ratio (NTR). The 28 dynamic signals for the linear microphone array were fed through high pass (100 $\mathrm{Hz})$ and low pass $(102,300 \mathrm{~Hz})$ signal conditioning filters from Precision Filters Inc. and into 4 National Instruments NI-PXI 6143 simultaneous sampling multifunction DAQ boards. Data were sampled at $210 \mathrm{kHz}$ with $60 \mathrm{data}$ averages, resulting in a 4096 point spectrum with a frequency resolution of $25.63 \mathrm{~Hz}$. Microphone actuator and freefield response corrections were applied, and background noise subtraction and shear layer corrections using Amiet's method ${ }^{12}$ were applied if the flight stream was operating. Far-field spectral levels were propagated to a common 1-foot arc, and frequencies were nondimensionalized to Strouhal number according to

$$
S t=\frac{f}{\left(U_{j} / D_{j}\right)}
$$

Lastly, spectral levels were adjusted to correspond, first to a $1 \mathrm{~Hz}$ common bandwidth, and ultimately to a per unit Strouhal number basis to be consistent with the frequency nondimensionalization:

$$
S P L(d B \text { perunit } S t \#)=S P L+10 \log \left(\frac{U_{j} / D_{j}}{(\Delta f)}\right) .
$$

where $\Delta f$ represents the original frequency bandwidth. Although atmospheric attenuation corrections are also typically applied to the data, a malfunctioning humidity sensor prohibited the consistent application of this correction. Therefore, atmospheric attenuation was not applied. Without correction of acoustic data to lossless conditions, spectral levels obtained over significantly different ambient weather conditions will diverge with increasing frequency. However, for the comparative studies of this work, the focus is typically at frequencies below $10 \mathrm{kHz}$. To quantify the impact of atmospheric attenuation corrections at $10 \mathrm{kHz}$, experiments were examined for which reliable humidity information was available at vastly different weather conditions (January-May). Comparing the differences between any two of these cases before and after application of the atmospheric attenuation correction yielded a maximum deviation of $0.5 \mathrm{~dB}$ at $10 \mathrm{kHz}$ for the furthest microphone location for which spectra are shown in this work $\left(135^{\circ}\right)$. More common deviations were approximately $0.1 \mathrm{~dB}$ at $10 \mathrm{kHz}$.

The corrections described above have been applied to all single microphone data unless otherwise noted. The phased array microphone data, however, go through a more complex processing algorithm described in the next section.

\section{Phased Array}

The JEDA phased array data acquisition system was separate from that of LSAWT but linked in via LabVIEW software interfaces. The 44 channels (41 array microphones plus 3 wall microphones) were fed through high pass $(100 \mathrm{~Hz})$ and low pass $(100,000 \mathrm{kHz})$ Precision filters cards and into a National Instruments PXI-8110 embedded controller system. All channels were simultaneously acquired at $200 \mathrm{kHz}$ for approximately 1 minute, resulting in 1470 averages of a 4096 point spectrum with a frequency resolution of $24.41 \mathrm{~Hz}$. Several individual microphone autospectra are presented in this work, and the correction process of the previous section was applied in these cases. The JEDA microphone selected for the single microphone measurements was approximately 11.3 inches upstream of the array center, thus avoiding the tightly-packed center of the array where microphone-to-microphone interference can occur. Examples of the repeatability of both the linear and phased arrays are shown in Figure 7 for the conditions shown. A schematic of the azimuthal orientation of the twin jet model with respect to each microphone array is shown in each respective plot. The variability in the linear array microphone of Fig. 7a is typically within $0.5 \mathrm{~dB}$ while the JEDA array microphone shown in Fig. 7b, with substantially more averages, exhibits less variability. 


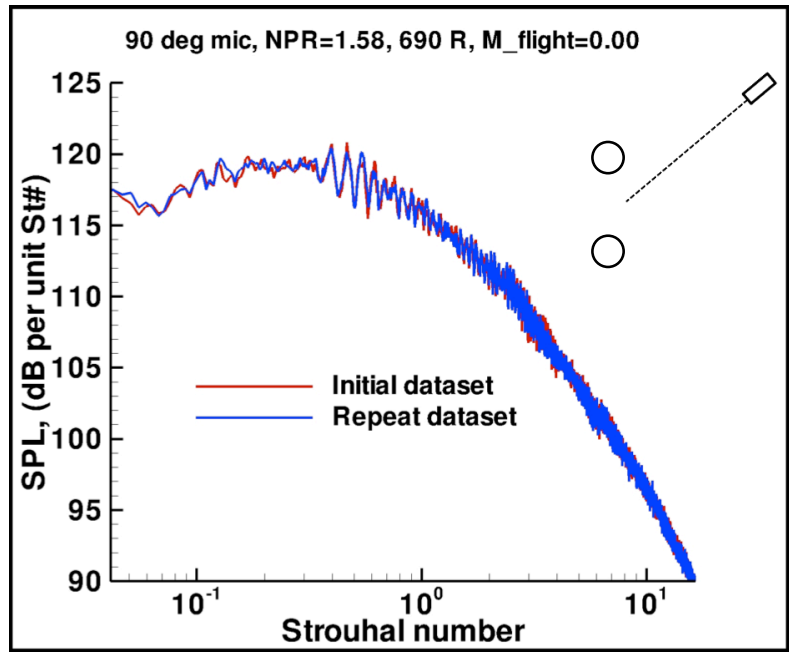

a)

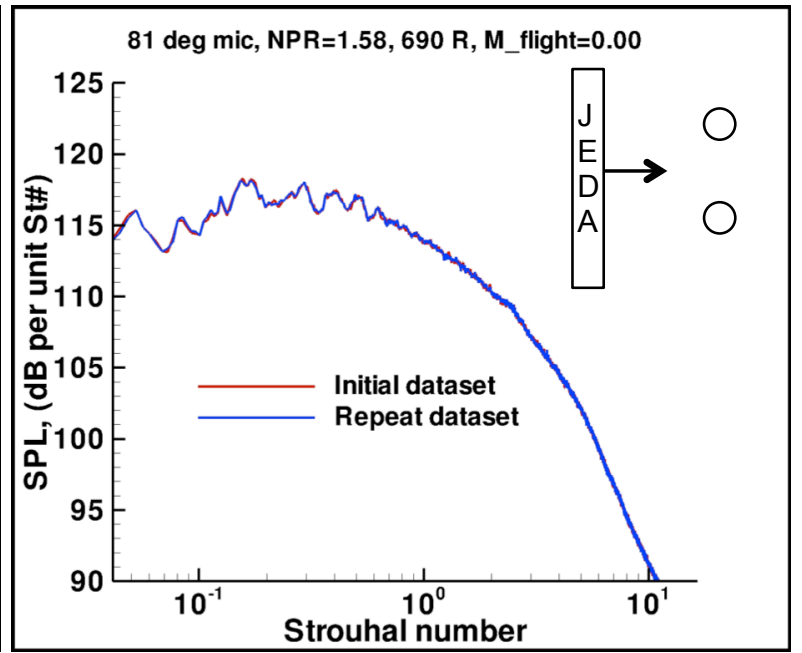

b)

Figure 7. Repeatability of a) linear array microphone at $90^{\circ}$ and b) JEDA single microphone at $81^{\circ}$. Azimuthal orientation is out-of-plane with respect to the JEDA.

The phased array microphone data can also combined to form a 41 element $\mathrm{x} 41$ element cross spectral matrix (CSM). This CSM can be used for standard array beamforming with the output power spectrum of the array described by Brooks and Humphreys ${ }^{10}$ as

$$
Y(\hat{e})=\frac{\hat{e}^{T} \hat{G} \hat{e}}{m_{0}^{2}},
$$

where $\hat{G}$ denotes the CSM, $Y(\hat{e})$ is a mean-pressure-squared per frequency bandwidth quantity, $m_{o}$ refers to the number of array microphones, and $\hat{e}$ refers to the steering vector matrix

$$
\hat{e}=\left[\begin{array}{c}
e_{1} \\
e_{2} \\
\cdots \\
e_{m_{0}}
\end{array}\right]
$$

with the component for each microphone $m$ as

$$
e_{m}=a_{m} \frac{r_{m}}{r_{c}} \exp \left\{j 2 \pi f \tau_{m}\right\}
$$

Here $f$ is frequency, $a_{m}$ is the refraction amplitude correction, $\boldsymbol{\tau}_{\boldsymbol{m}}$ is the time required to propagate from grid point $n$ to the microphone $m$, and $r_{m}$ and $r_{c}$ refer to the distances to the microphone and the center microphone respectively, in emission coordinates.

The resulting beamform maps are informative, but they are dependent upon the array size and geometry. Therefore, the Deconvolution Approach for the Mapping of Acoustic Sources (DAMAS) ${ }^{10}$ is employed to remove the beamforming characteristics from the result. In this case a modeled cross-spectral matrix is considered in place of $\hat{G}$ in Eq. (3) leading ultimately to an iterative algorithm to solve the inverse problem. This technique has been successfully applied to single- and dual-stream jet flows by Brooks, et al. ${ }^{11}$, and the lessons shared in their work 
regarding microphone weighting and CSM phase conditioning due to the thick free stream shear layer are important to apply here.

Brooks and Humphreys ${ }^{10}$ use diagonal removal to reduce the impact of microphone self noise and microphone weighting to maintain beamform resolution over a range of frequencies. This methodology is also employed in the current work and alters the beamform according to

$$
Y(\hat{e})=\frac{\hat{e}^{T} \hat{W} \hat{G}_{\text {diag }=0} \hat{W}^{T} \hat{e}}{\left(\sum_{m=1}^{m_{0}} w_{m}\right)^{2}-\left(\sum_{m=1}^{m_{0}} w_{m}\right)}
$$

Eq. (6) shows $\hat{W}$ as a row matrix containing the microphone weighting functions $w_{m}$. For a more recent DAMAS application to jet noise, Brooks, et al. ${ }^{11}$ found a linear weighting to effectively reduce sidelobes and increase signalto-noise ratio:

$$
w_{m}=w_{1}+\left(1-w_{1}\right)\left(\frac{R_{m}}{R_{m_{0}}}\right) \text {, }
$$

in which $R_{m}$ is the radial distance from the JEDA center microphone to microphone $m, R_{m_{0}}$ is the outer ring radius ( $R_{m_{0}}=24$ inches), and $w_{m}=0.1$.

While the DAMAS technique accounts for propagation through thin shear layers using the method of Amiet ${ }^{12}$, Brooks, et al. ${ }^{11}$ found that the thick shear layers in the LSAWT required further consideration. This led to the formulation of a CSM phase conditioning method using measured acoustic data from a point source at a known location. With this method, the time delay between microphone pairs, $\Delta \tau_{i j}$, is averaged over a range of frequencies, weighted by coherence $\gamma_{i j}^{2}(f)$ :

$$
\Delta \bar{\tau}_{i j}=\frac{\sum_{n=n_{l}}^{n=n_{u}} \Delta \tau_{i j}(f) \gamma_{i j}^{2}(f) \Delta f}{\sum_{n=n_{l}}^{n=n_{u}} \gamma_{i j}^{2}(f) \Delta f} .
$$

Brooks, et al. ${ }^{11}$ typically used a lower frequency limit of $5000 \mathrm{~Hz}$ and an upper frequency limit where the coherence $(10 \log )$ is maintained at or above $-20 \mathrm{~dB}$. Note that $n_{u}$ and $n_{l}$ represent the lower and upper bin numbers where $f=n$ $\Delta f, \mathrm{n}=0,1,2, \ldots$ The average phase angle is then

$$
\left(\bar{\varphi}_{i j}\right)_{\text {point source }}=2 \pi f \Delta \bar{\tau}_{i j}
$$

and a measured CSM can be conditioned according to

$$
\left(G_{i j}\right)=\left|G_{i j}\right| \exp j\left\{\varphi_{i j}+\left(\varphi_{i j}^{\prime}-\bar{\varphi}_{i j}\right)_{\text {point source,Tpoint source }} \sqrt{T_{\text {point source }} / T_{\text {test }}}\right\}
$$

Eq. (10) shows the measured phase $\varphi_{i j}$ for the test case is adjusted based on an error offset between the predicted and average measured phase of the point source $\left(\varphi_{i j}^{\prime}-\bar{\varphi}_{i j}\right)$ and further corrected for any ambient temperature differences between the test and point source cases. The CSM conditioning methodology outlined here corrects for unexpected phase differences due, not only to propagation through a thick shear layer, but also to simple array positioning error.

These methods are employed in the current DAMAS processing in a manner consistent with Brooks, et al. ${ }^{11}$ in which 7 FFT bins of $24.4 \mathrm{~Hz}$ each are vectorially added to generate a $171 \mathrm{~Hz}$ bandwidth "narrowband" frequency.

American Institute of Aeronautics and Astronautics 
These frequency bands are then summed in the appropriate third-octave band ranges. Although several third-octave bands were investigated, the current work will focus on a mid-range band of $6300 \mathrm{~Hz}$. This band is chosen because it is low enough to be close to the spectral region where changes are most noticeable in the comparisons, yet high enough to usually avoid displaying strong coherent source behavior for which this version of DAMAS is not suited.

An example without flow is shown in Fig. 8 in which two incoherent broadband point sources are placed along the model centerline between jets with one source at the nozzle exit plane ( $x=79$ inches) and the second source 40 inches downstream. The JEDA array is located at $90^{\circ}$ to the model system. The standard beamform result is shown on top, and the DAMAS-processed figure is shown on the bottom for the $6300 \mathrm{~Hz}$ third-octave band. The inner diameters of the point source tubes are indicated by the circles. The improved resolution of the deconvolution approach is evident compared to the beamform result. The CSM conditioning approach utilizes the upstream point source, thus, the slight displacement of the downstream source could be due to an error in source placement. Unless otherwise noted, all DAMAS results shown in the current work use 250 iterations of the deconvolution algorithm.

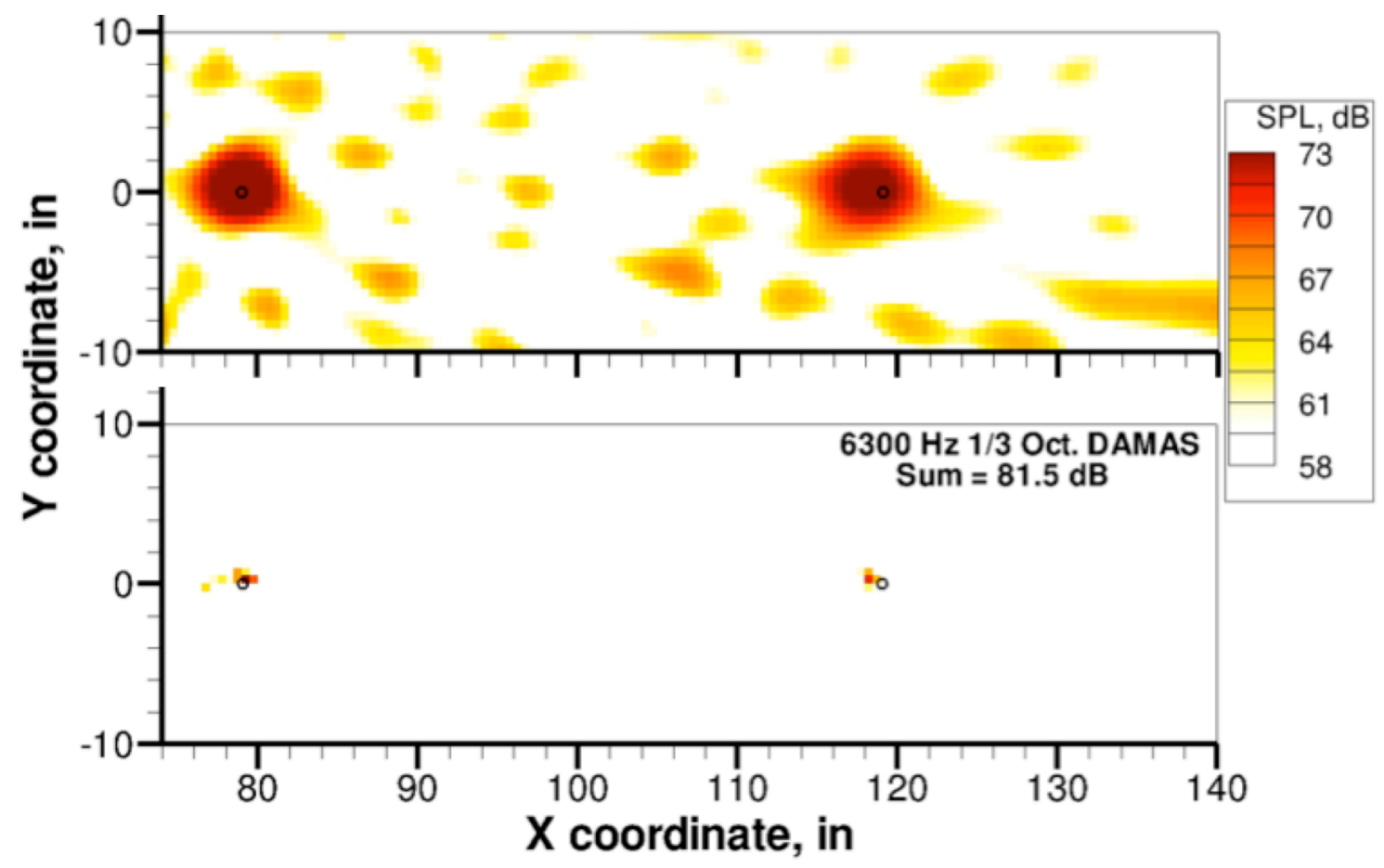

Figure 8. Calibration point source results at $6300 \mathrm{~Hz}$ third-octave band for two point sources at (79", 0") and (119”, 0") with no jet or wind tunnel flow. Beamforming result (top) and DAMAS result using CSM conditioning and 250 iterations (bottom).

Figure 9 shows a single point source with wind tunnel flow at $M_{W T}=0.1$. The top contour plot shows the DAMAS result without CSM conditioning while the bottom plot shows the DAMAS result with CSM conditioning. Although there is slightly more scatter for this particular CSM conditioning case, the source is better positioned with CSM conditioning, which is used throughout the remainder of the work. Note that a small scan grid plane of higher resolution is utilized for this study compared to the two point sources of Fig. 8. The grid resolution is $0.25 \times 0.25$ inches, and the magnitude of each color square in the contour corresponds to the level at the bottom left corner coordinates of each square. This increased grid resolution is used unless investigations at $x / D>10$ are required. Lastly, the sum value in the DAMAS contour plots refers to the integrated SPL for the given third-octave band over the source region. 


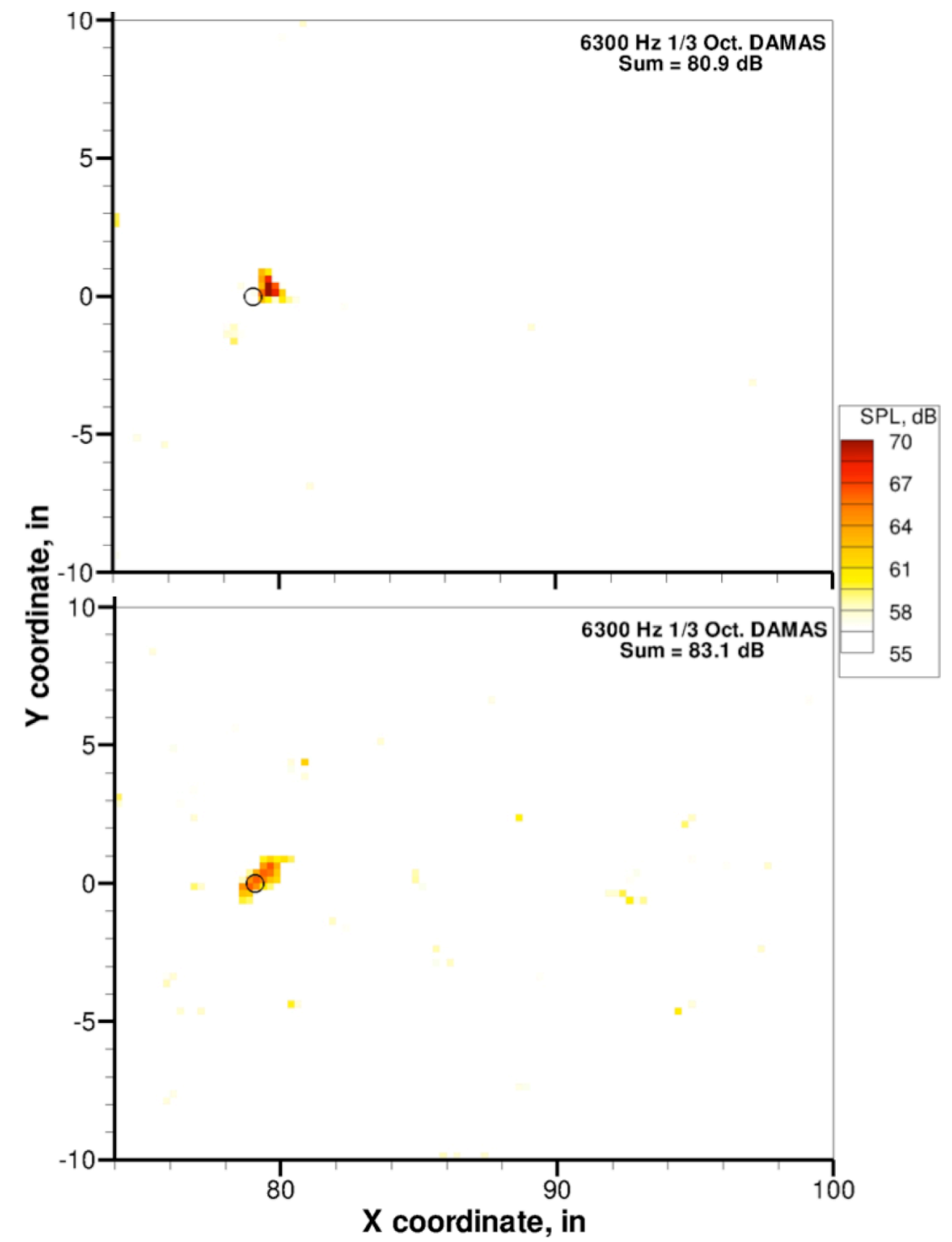

Figure 9. Calibration point source results at $6300 \mathrm{~Hz}$ third-octave band for one point source at (79", 0") with $M_{W T}=0.1$. No CSM conditioning in top figure, with CSM conditioning in bottom figure. 


\section{Experimental Results}

\section{A. Flow Conditions}

The majority of flow conditions examined in this work focus on the takeoff cycle of the HWB that will ultimately be simulated using two dual-stream CJES units. However, the present twin jet model has only singlestream jets. Therefore, mass-weighted mixed temperatures and velocities that would be expected as the CJES fan and core streams mix are typically used here to establish the setpoints for the twin jet model. It should be noted that the fan flowfield is set here to the same conditions as the wind tunnel flight stream to minimize flow separation off the model. Table 1 shows typical flow conditions used in this work with the HWB takeoff (mixed flow) cycle shown in the first row.

Table 1. Typical experimental conditions assuming standard day conditions.

\begin{tabular}{|c|c|c|c|c|c|}
\hline $\boldsymbol{N P R}_{\text {core }}$ & $\boldsymbol{N T R}_{\text {core }}$ & $\boldsymbol{M}_{\text {acoustic }}$ & $\boldsymbol{f}_{\boldsymbol{c}}\left(=\boldsymbol{U}_{\boldsymbol{j}} / \boldsymbol{D}_{\boldsymbol{j}}\right)(\mathbf{H z})$ & $\boldsymbol{M}_{\text {wind tunnel }}$ & $\boldsymbol{F a n}$ Stream \\
\hline 1.58 & 1.33 & 0.90 & 6045 & $0.0,0.22$ & Matches flight stream $P$ and $T$ \\
\hline 1.80 & 1.84 & 1.19 & 7989 & 0.10 & Matches flight stream $P$ and $T$ \\
\hline 1.88 & 2.37 & 1.40 & 9362 & 0.10 & Matches flight stream $P$ and $T$ \\
\hline
\end{tabular}

\section{A. Mean Flowfield Measurements}

The mean flowfield properties of the jet plume when using either the straight adapters or the $5^{\circ}$ angle adapters are shown at several axial stations in Figs. 10 and 11. Figure 10 shows the Mach number profiles for the mixed flow condition representative of the HWB cycle at takeoff: $N P R=1.58, T_{T}=230^{\circ} \mathrm{F}$, and flight stream Mach number, $M_{W T}=$ 0.22 . While the straight twin jets show little evidence of merging before $x / D=14$, the $5^{\circ}$ canted twin jets merge much more quickly, before $x / D=10$. It should be noted that the centerline-to-centerline spacing for the straight adapter case is 3.23 jet diameters, approximately the same spacing anticipated for the HWB test. However, in order to properly align the twin jets when using the $5^{\circ}$ angle adapters, the centerline-to-centerline spacing had to change slightly to 3.12 diameters. This spacing difference, however, is far outweighed by the effect of the $5^{\circ}$ cant angle. Figure 11 shows the analogous total temperature maps normalized by the charging station total temperature. Once again, the canted twin jets show evidence of merging several diameters upstream of the straight twin jets. The flight stream surrounding the canted jets is at a lower temperature ratio than the straight jet cases. This is due to the difference in ambient temperature between these two sets of data taken approximately two months apart. It is also interesting to note that as the canted jets merge, the predominant plume shape tends to be an oval with the major axis oriented in the vertical direction, not in the horizontal direction, as one might expect.

A more quantitative location of the merging point for each case can be seen in Fig. 12 in which the total pressure is measured along the centerline between nozzles as the rake is traversed downstream. The total pressure is normalized by the static pressure within the wind tunnel flow and plotted versus $x / D$. In both cases the total pressure first drops slightly with increasing downstream distance, likely due to wake effects of the model. Then as the jet shear layers begin to reach the centerline location, the total pressure increases. This increase first occurs at approximately $5.75 x / D$ for the canted jets and $11 x / D$ for the straight jets. Thus, the $5^{\circ}$ canted jets merge almost twice as quickly as the straight jets. The shear layer spreading angle corresponding to the straight jets, based on these measurements is $5.8^{\circ}$. One might expect the canted jets to merge at an angle equal to the shear layer spreading angle plus the cant angle, or approximately $10.8^{\circ}$. Indeed, the canted jets do merge at an equivalent angle of approximately $10.4^{\circ}$. The maximum centerline total pressure occurs at $15 x / D$ for the canted jets while the more gradual pressure rise with the straight jets is still observed at the $25 x / D$ measurement station. 


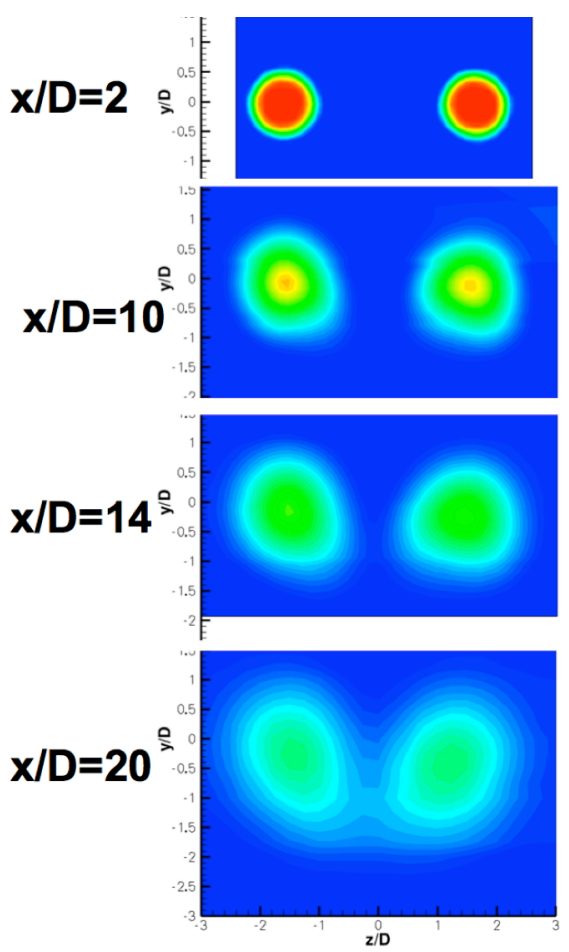

Straight Adapters
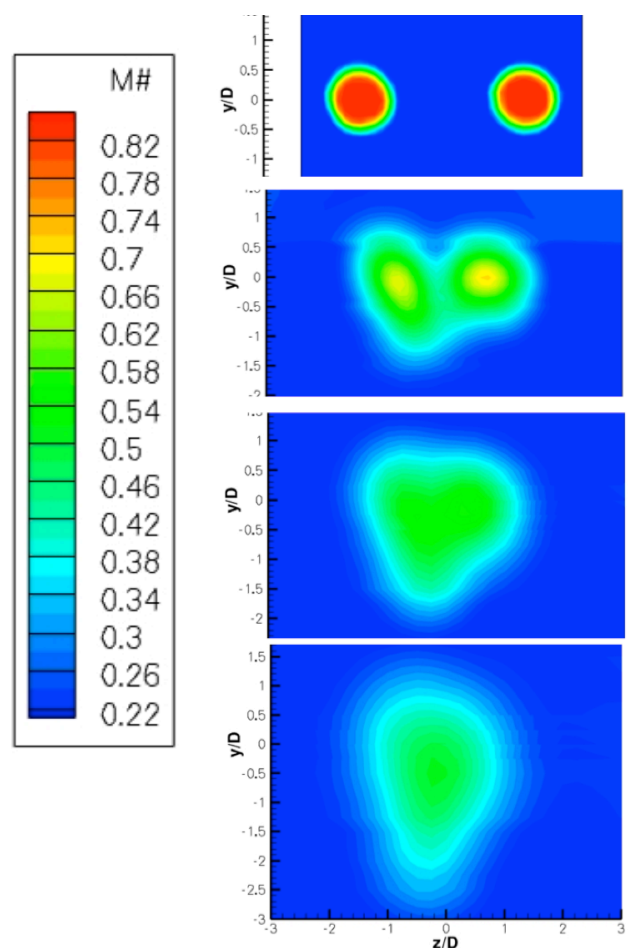

5 Degree Angle Adapters

Figure 10. Mach number maps comparing straight adapters with $5^{\circ}$ angle adapters at four different axial stations for mixed flow takeoff condition $\left(N P R=1.58, T_{T}=230^{\circ} \mathrm{F}, M_{W T}=0.22\right)$.

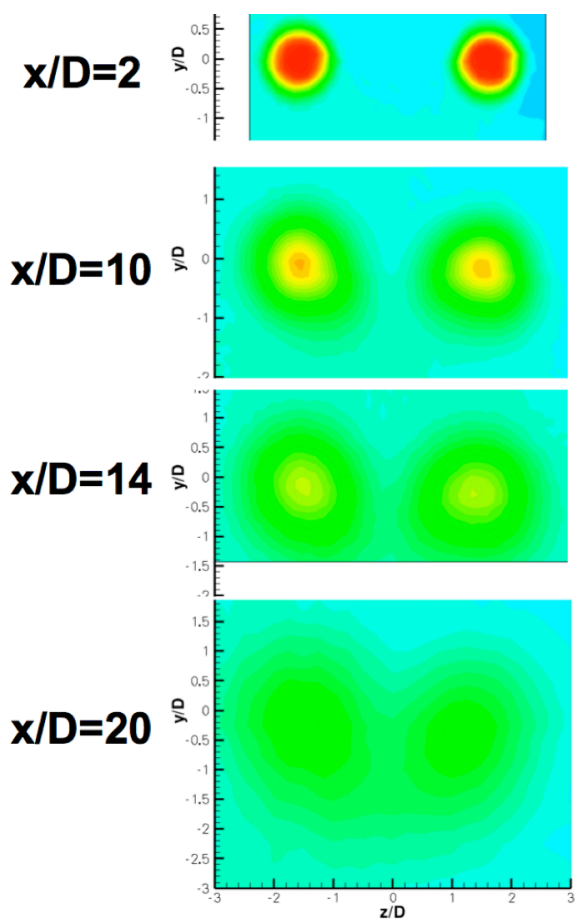

Straight Adapters
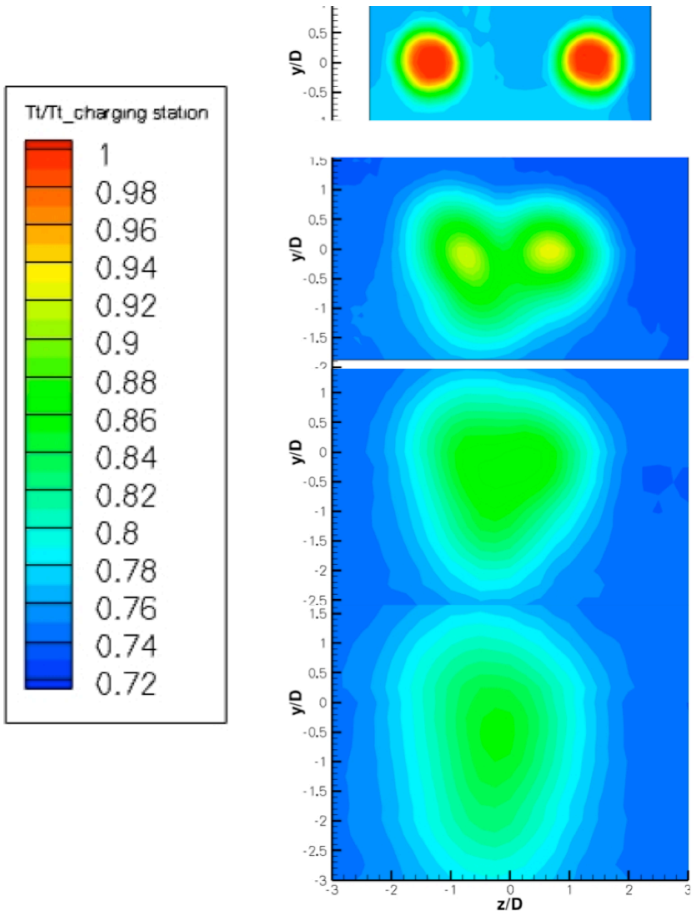

5 Degree Angle Adapters

Figure 11. Total temperature maps comparing straight adapters with $5^{\circ}$ angle adapters at four different axial stations for mixed flow takeoff condition $\left(N P R=1.58, T_{T}=230^{\circ} \mathrm{F}, M_{W T}=0.22\right)$. 


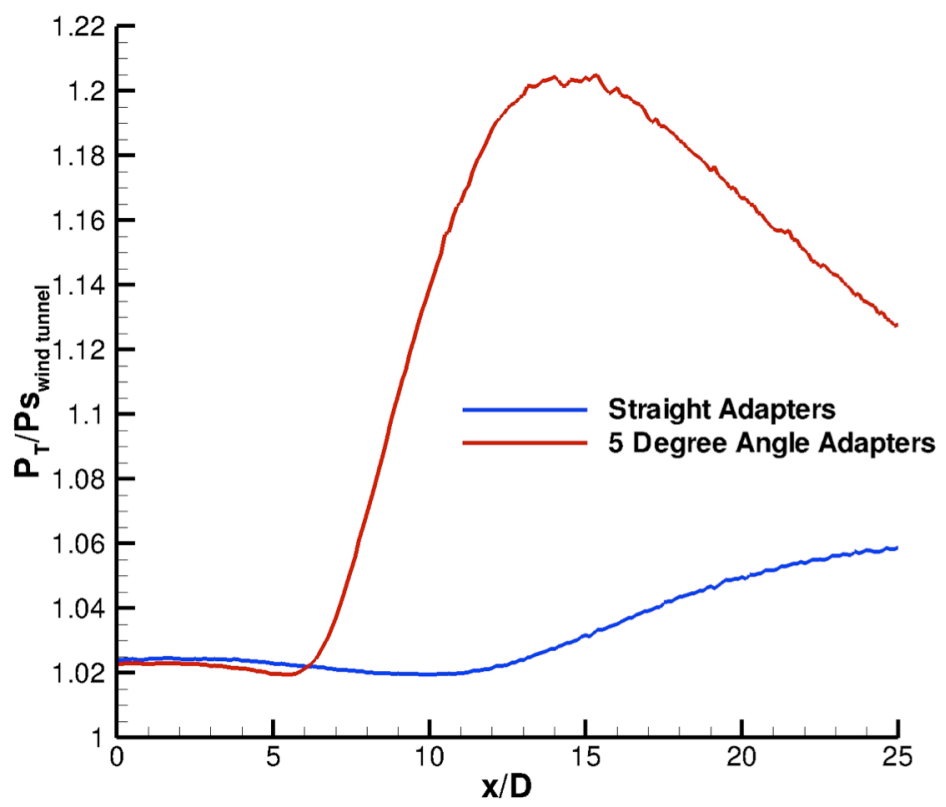

Figure 12. Pressure plot comparing straight adapters with $5^{\circ}$ angle adapters along the model centerline between the two nozzles for mixed flow takeoff condition $\left(N P R=1.58, T_{T}=230^{\circ} \mathrm{F}, M_{W T}=0.22\right)$.

\section{B. Acoustic Measurements}

\section{Twin Jet Interactions}

Before considering the specific HWB application it is useful to consider the acoustic effect of two closely spaced jets as discussed in Section I. Far-field acoustic spectra of the twin jet model with varying nozzle spacings are measured using the linear acoustic array. The model system is oriented in the out-of-plane, or flyover, configuration with respect to the microphone array. At this azimuthal orientation one would expect minimal jet-jet shielding. Figure 13 shows the results at $90^{\circ}$ and $135^{\circ}$ with respect to the jet inlet axis as well as the directivity. Four increasing spacings are shown as well as the single jet $+3 \mathrm{~dB}$ case, which is considered the representative baseline noise level expected if the jets did not affect each other's flowfield or acoustic source propagation. It should be noted that the single jet measurements are obtained by blanking off the starboard jet at the adapter and using a cone fairing to avoid flow separation at the blank flange. Figure 13a shows little effect of nozzle spacing on spectral levels (the high frequency deviations observed at $\mathrm{St}>2$ are due to differences in atmospheric attenuation for different test conditions). However, Fig. 13b shows an impact of spacing further downstream at $135^{\circ}$ from the jet inlet. The two closest spacings show higher peak noise levels than the single jet $+3 \mathrm{~dB}$ case with $S / D=2.62$ showing slightly higher levels than $S / D=3.23$. The two largest spacings exhibit a different spectral shape with an increase at lower Strouhal numbers and a flattening at the peak. This behavior is similar to that observed by Bozak and Henderson ${ }^{9}$ in which a secondary peak started to emerge at large nozzle spacings. Figure 13c shows the directivity patterns for these same cases. While there are some slight increases with the twin jet cases at the far upstream angles to the jet axis (below $60^{\circ}$ ), the more noticeable effect is that the OASPL levels grow more quickly for the twin jet cases as one approaches the jet exit axis. Not surprisingly, as the jet spacing increases the OASPL increase is seen at further aft angles, as the jets take more axial distance to merge. For each twin jet case, the maximum OASPL, when considering all angles, is greater than the single jet equivalent maximum OASPL by approximately 2 dB. 


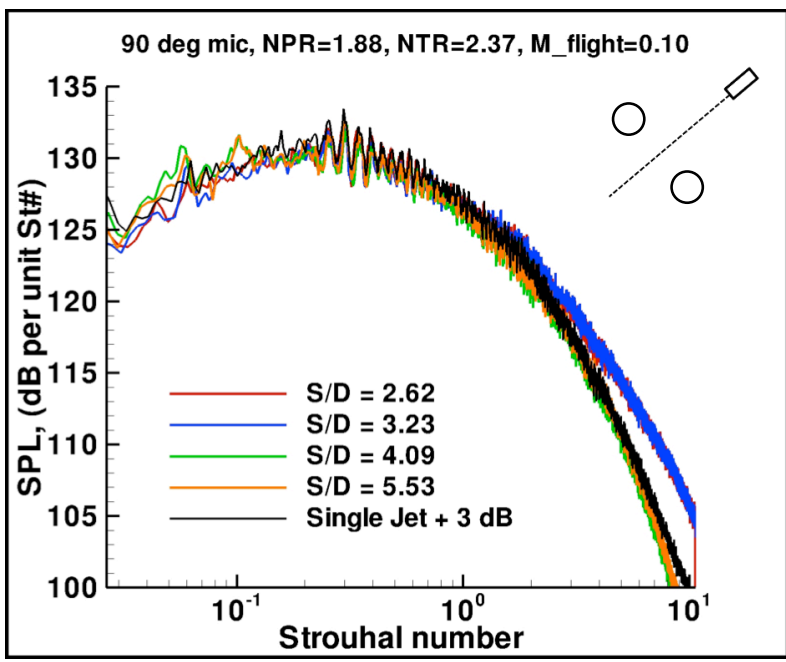

a)

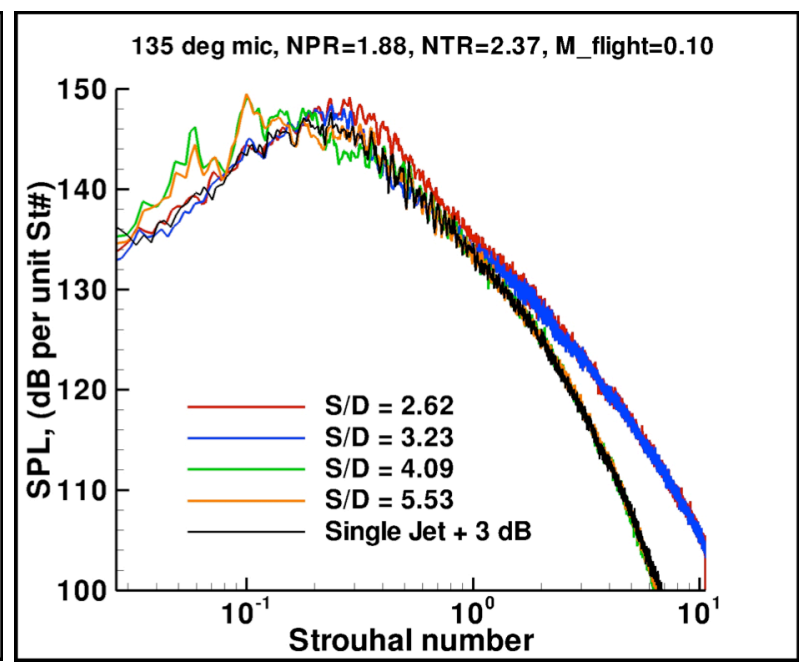

b)

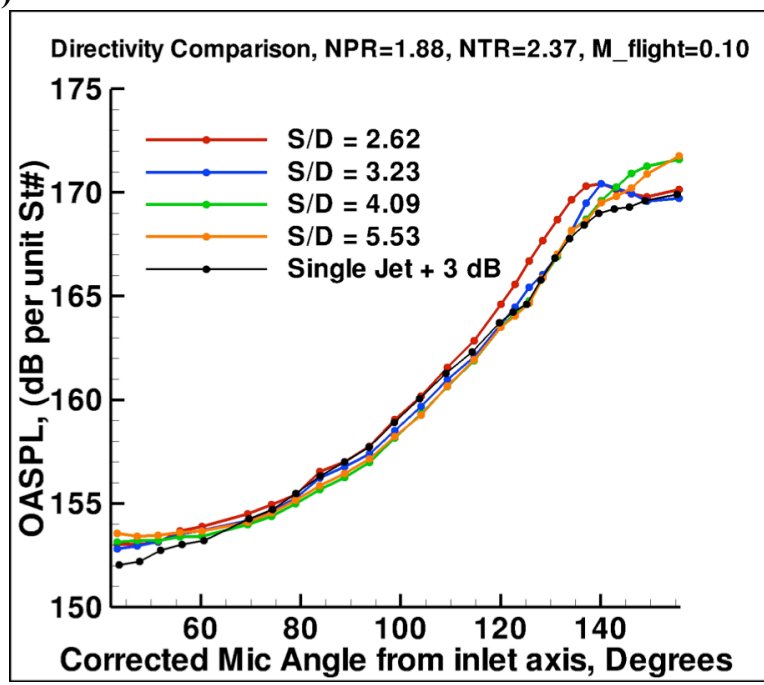

c)

Figure 13. Linear array measurements of jet spacing effect in out-of-plane configuration a) $90^{\circ}$ spectra, b) $135^{\circ}$ spectra, and c) directivities.

\section{Canted Jets}

The noise increases observed in the out-of-plane azimuthal direction for twin jets have all been for cases in which the jets are parallel to one another. Another consideration is for twin jets canted inward toward each other. This configuration is relevant to potential HWB designs with over-the-wing engine mounts because the inward canted jets allow the nacelle inlets to be canted outward, maintaining better alignment with the flowfield over the wing surface and, thus, reducing inlet distortion. Although there would be a performance penalty due the canted thrust vector, the performance benefit of the reduced inlet distortion could be enough to warrant its consideration.

As shown in Section IIIA, a set of $5^{\circ}$ adapters can replace the straight adapters in the twin jet model system. This permits comparisons between canted and straight jets at different jet centerline spacings. Figure 14 shows spectral comparisons at $81^{\circ}$ and $129^{\circ}$ with the twin jet model system oriented both out-of-plane and in-plane with respect to the phased array. The spectra from a single microphone of the JEDA array are shown. As mentioned in Section IIC, the microphone selected is approximately 11.3 inches upstream of the center of the array to minimize potential microphone-to-microphone interference near the tightly packed array center. The baseline HWB configuration of $S / D=3.23$, using $0^{\circ}$ adapters is compared to two spacings with $5^{\circ}$ inward cant angle, as well as the single jet $+3 \mathrm{~dB}$ case. (The baseline HWB configuration and the $S / D=3.12,5^{\circ}$ inward cant angle case are the same configurations for which mean flowfield data are shown in Figs. 10-12.) In Figs. 14a and $14 \mathrm{~b}$ the $5^{\circ}$ inward cant angle cases show slightly lower noise levels than the parallel flow case. In particular, for the $5^{\circ}$ case with $S / D=2.27$ 


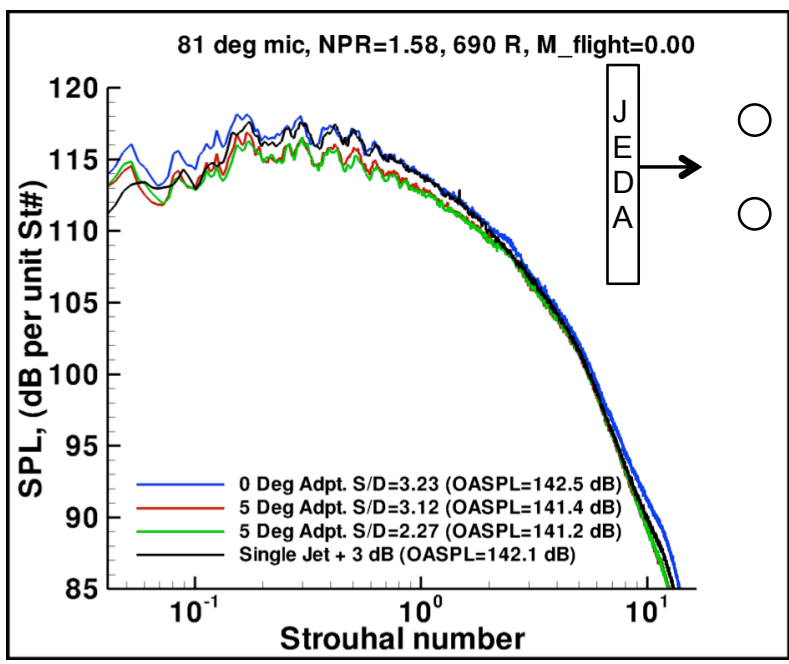

a)

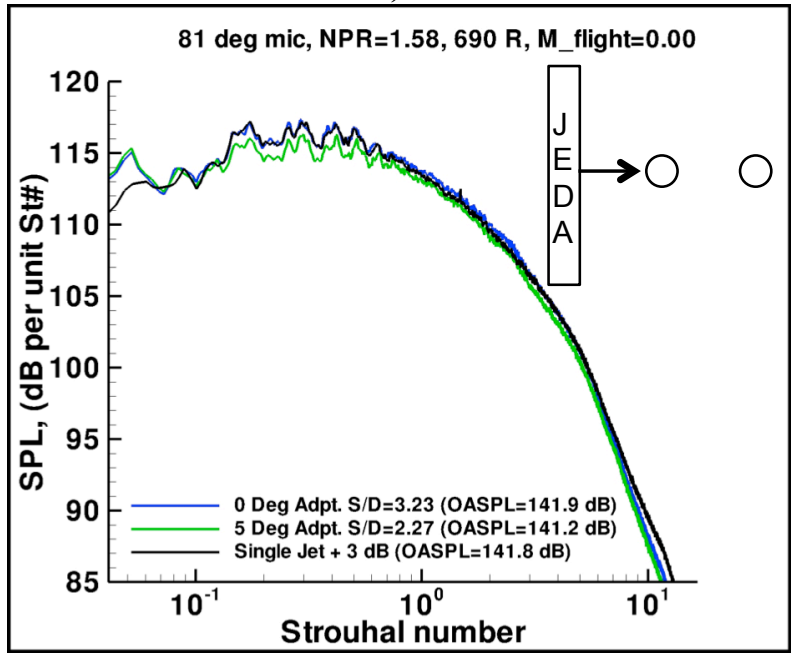

c)

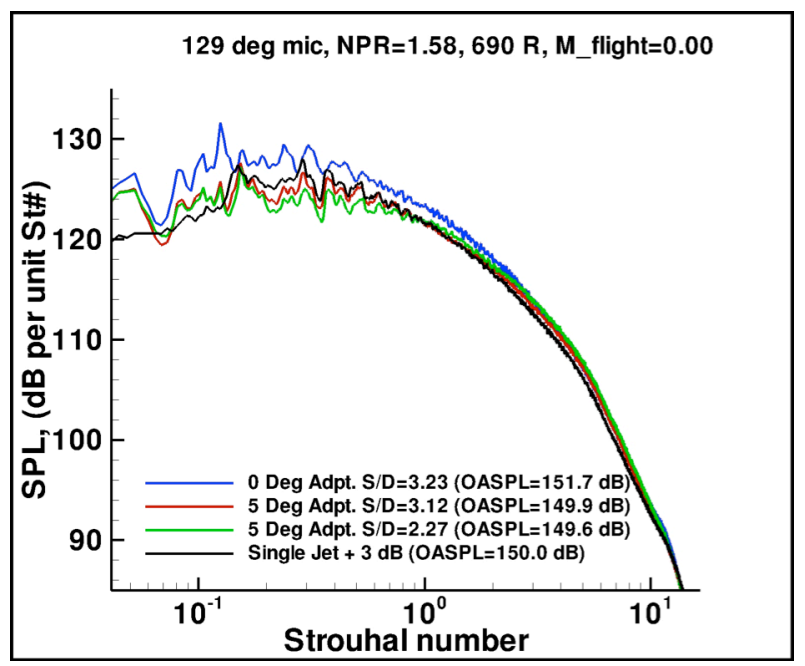

b)

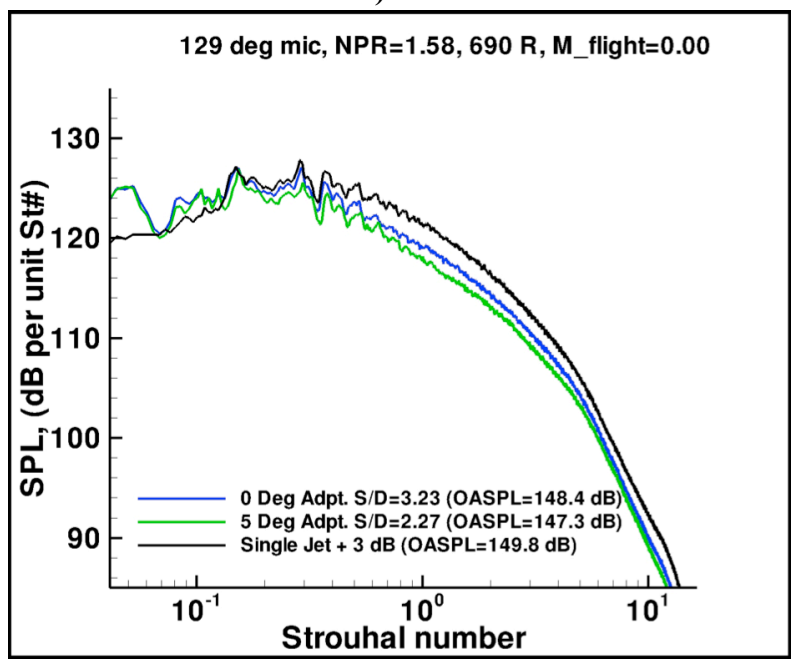

d)

Figure 14. JEDA microphone spectra showing the acoustic impact of canted jets for the out-of-plane configuration at a) $81^{\circ}$ and b) $129^{\circ}$, and for the in-plane configuration at c) $81^{\circ}$ and d) $129^{\circ}$.

the spectra and OASPL are lower than the parallel flow case by 1.3-2.1 dB and also slightly lower than the single jet $+3 \mathrm{~dB}$ case. This would suggest that the typical out-of-plane noise increases observed in twin jet configurations are mitigated by the more intense interaction of closely-spaced canted jets. Figures $14 \mathrm{c}$ and $14 \mathrm{~d}$ show in-plane shielding and the additional benefit of the closely-spaced canted jets, particularly at the downstream angle in Fig. $14 \mathrm{~d}$ where a $2.5 \mathrm{~dB}$ reduction in OASPL with respect to the single jet $+3 \mathrm{~dB}$ case is noted. Similar reductions were also observed for other setpoints including forward flight simulation.

Phased array measurements are used to further investigate the acoustic effects of the canted jets in Fig. 15 . The DAMAS result for the baseline HWB configuration is shown at the top of Fig. 15 , compared to the $S / D=2.27,5^{\circ}$ inward cant angle at the bottom of Fig. 15. The noise map of the canted jets distinctly indicates quick merging. Furthermore, the amplitude of the source strengths in the inner shear layers of the parallel twin jets seem to be reduced when the jets merge in the canted configuration. This is likely due to intense mixing that takes place among the shear layer structures. The more fractured appearance of the noise maps further downstream is possibly due to coherence over the $6300 \mathrm{~Hz}$ third-octave band. Because the DAMAS approach used here assumes completely incoherent noise sources, when a significant level of coherence is encountered, the noise maps often appear fractured, as Brooks, et al. ${ }^{11}$ observed. 


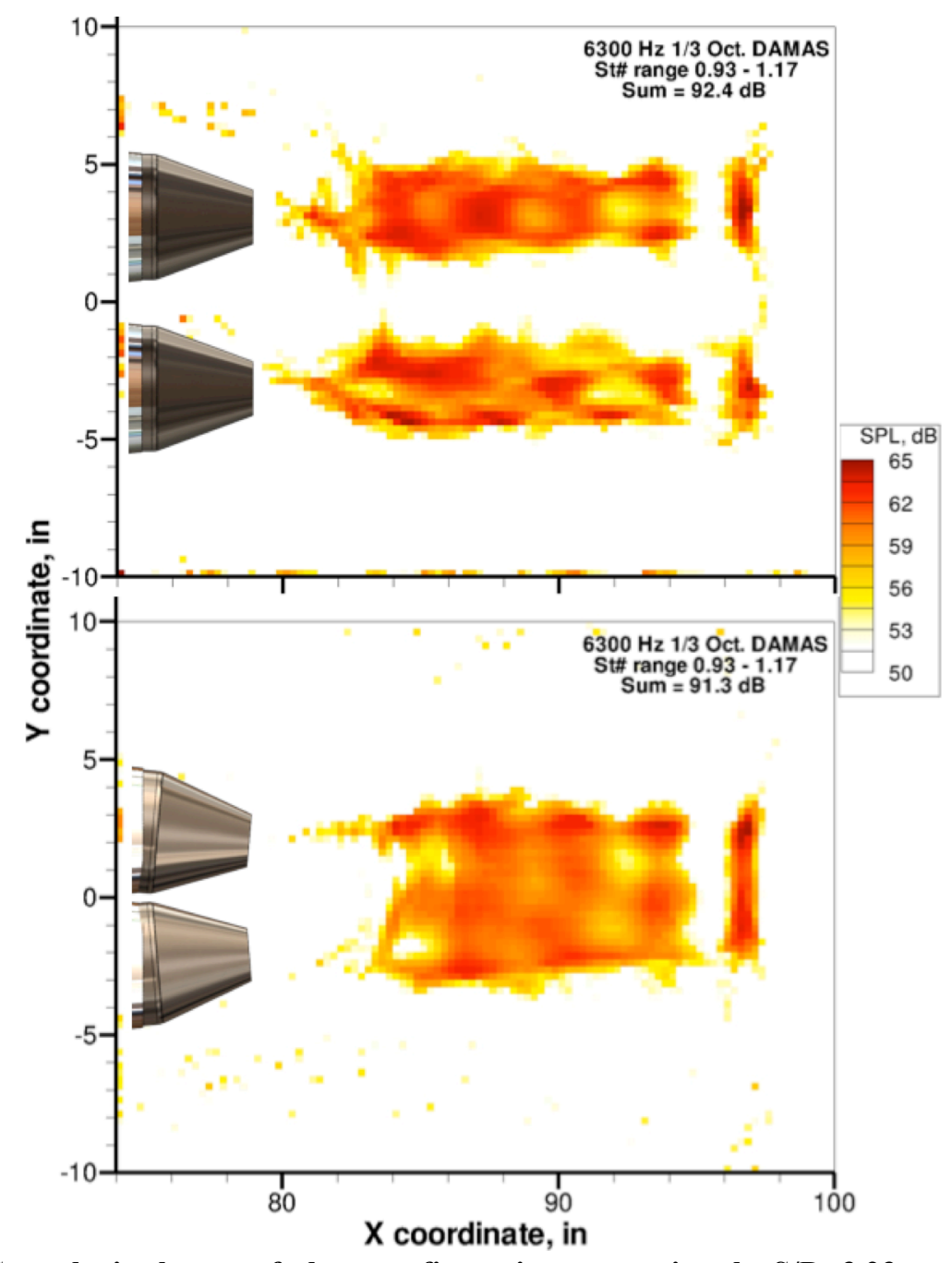

Figure 15. DAMAS results in the out-of-plane configuration comparing the $S / D=3.23$ spacing with $0^{\circ}$ adapters (top) to the $\mathrm{S} / \mathrm{D}=\mathbf{2 . 2 7}$ spacing with $5^{\circ}$ adapters canting the jets toward each other (bottom). Flow conditions are identical to those of Fig. 14.

\section{Elevon Deflection}

The remainder of the results involve the use of the 1.9\% HWB aft-deck fuselage model. Figure 16 shows the details of this geometry as it is installed with respect to the twin jet model. The twin jets are at the HWB spacing (3.23 D, where D is the diameter of the fan stream in the case of the HWB) and positioned as closely as possible to the intended HWB baseline axial and vertical locations. The axial distance from the jet exit plane to the model trailing edge along the jet centerline is $2.8 \mathrm{D}$ as shown in Fig. 16a, and the vertical distance from the jet centerline to the fuselage surface at the jet exit plane is approximately 1.3 D. A challenge often encountered with jet shielding studies is to position the shield close enough to the jet flow, given the typical closeout angle of the jet rig and nozzle. In this case support rails are attached to an upstream mounting flange, and the aft deck model is curved upward to allow for close proximity to the twin jet model as evident in Fig. 16b. Nevertheless, to maintain capability to rotate the twin jet model system and adjust nozzle spacing, model clearance issues dictated a slight angular separation of approximately $6^{\circ}$ between the aft deck model and the nozzle centerline. Both aft deck model and the nozzles were rotated away from each other by about $3^{\circ}$ to accomplish this separation, as shown in Fig. 16c. Investigations of acoustic spectral levels with and without this rotation did not show significant differences. 


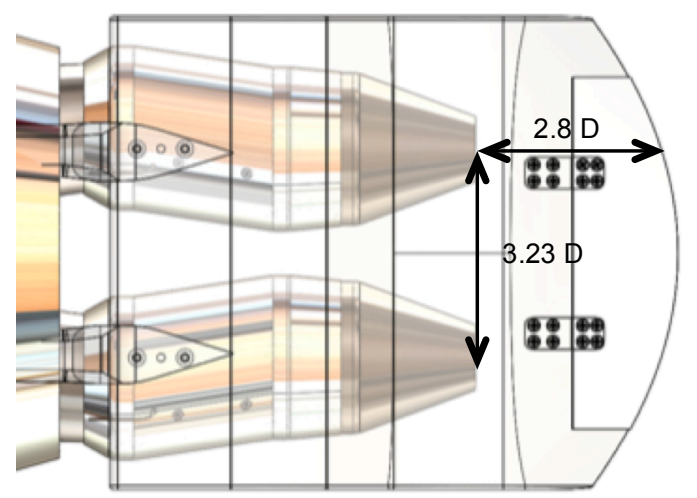

a)

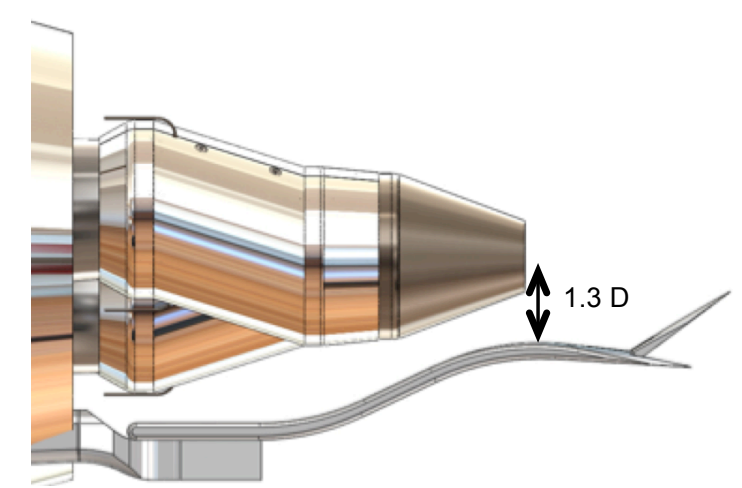

b)

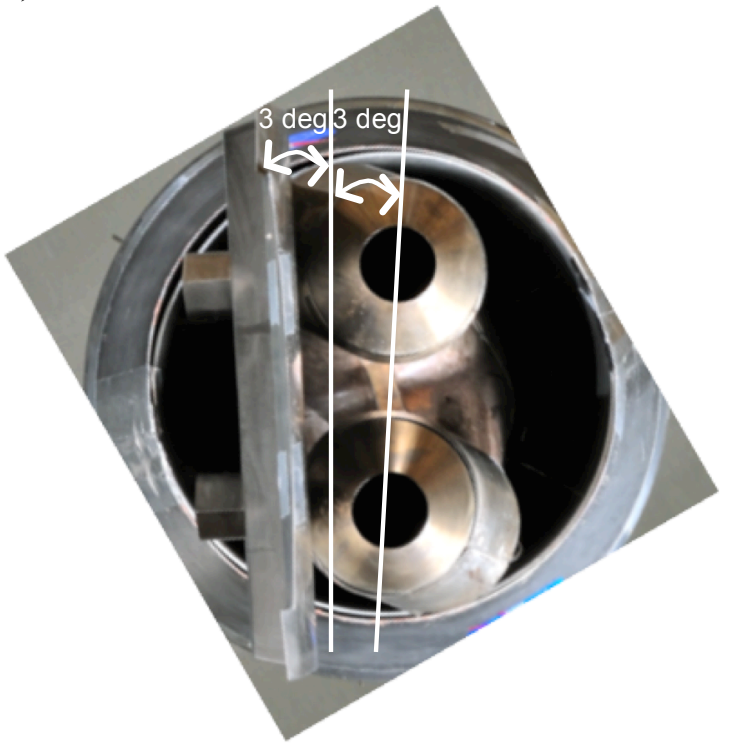

c)

Figure 16. HWB aft deck fuselage model installed with twin jet model in a) the out-of-plane configuration and $b$ ) the in-plane configuration with $40^{\circ}$ upward elevon deflection, as well as c) an end-on view showing slight model rotation necessary for clearance.

The acoustic impact of a $40^{\circ}$ elevon upward elevon deflection is investigated because the current HWB design requires such a deflection for rotation off the runway. The in-plane azimuthal orientation is considered here since the deflection is not anticipated in flight. This deflection, as seen in Fig. 16b does impinge into the jet plume. Single microphone spectral results from the individual JEDA microphone (with corrected position to account for shear layer refraction) are shown in Fig. 17 with and without the elevon deflected upward $40^{\circ}$. In Fig. $17 \mathrm{a}$ at $79^{\circ}$ the deflected elevon case shows a broadband spectral increase corresponding to $1.2 \mathrm{~dB}$ OASPL. However, further downstream at $129^{\circ}$ (Fig. 17b), slight increases at low frequency are essentially balanced by the shielding of higher frequencies such that virtually no OASPL increase is observed. Figure 17c shows directivity results from the linear array which is positioned at a $50^{\circ}$ elevation angle compared to the JEDA array. Large increases in noise greater than $5 \mathrm{~dB}$ are seen at the upstream angles, but the noise increase gradually diminishes until the deflected elevon levels are similar to or even lower than the undeflected elevon. These results suggest the noise changes for this particular setup are due primarily to reflection (upstream) and some shielding far downstream.

Phased array measurements of the elevon configuration at $0^{\circ}$ and $40^{\circ}$ upward deflection are shown in Fig. 18. With the scan plane aligned with the port jet (closer to array) centerline, the $6300 \mathrm{~Hz}$ third-octave band DAMAS results are shown for both configurations. The increased noise at the deflected elevon trailing edge is evident in the bottom contour of Fig. 18, as is the reflected noise in the upstream direction. The integrated source map also shows a $1.2 \mathrm{~dB}$ increase in OASPL, consistent with the increase observed for the $79^{\circ}$ single microphone measurement. 


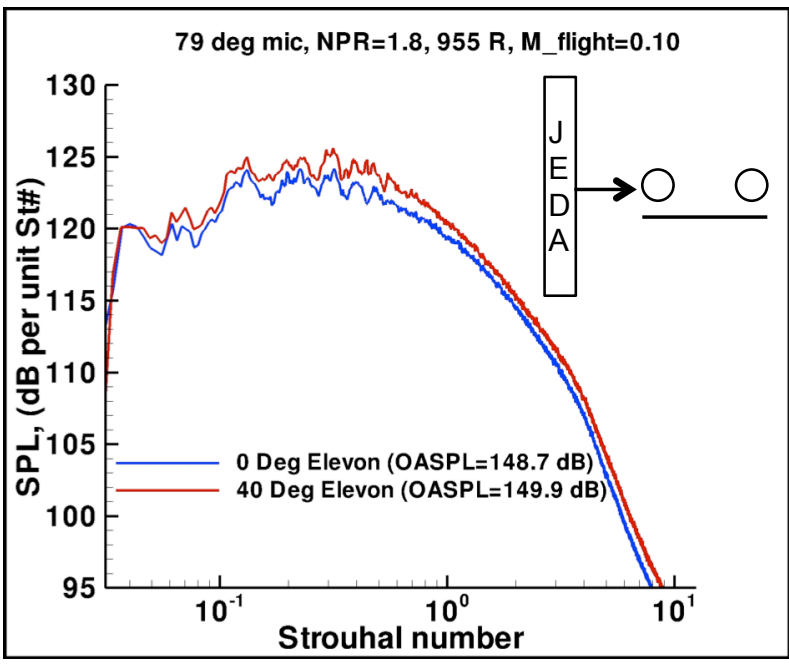

a)

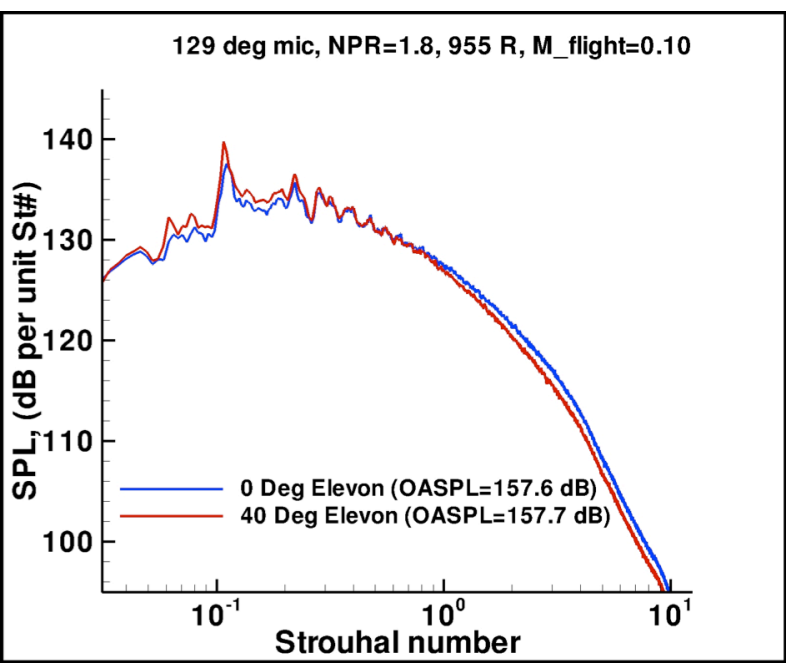

b)

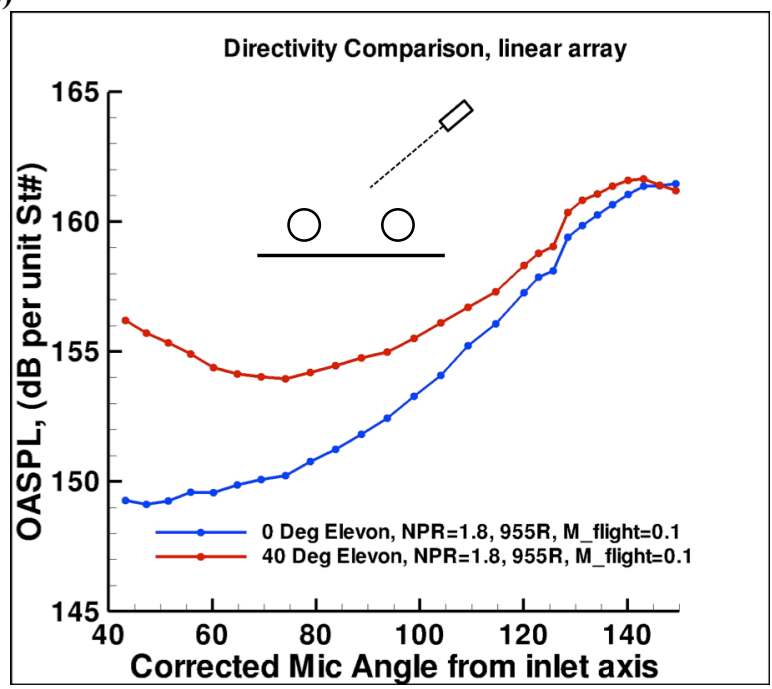

c)

Figure 17. JEDA microphone spectra showing the acoustic impact of elevon deflection for the in-plane configuration at a) $79^{\circ}$, b) $129^{\circ}$, and c) corresponding directivities from the linear array. 


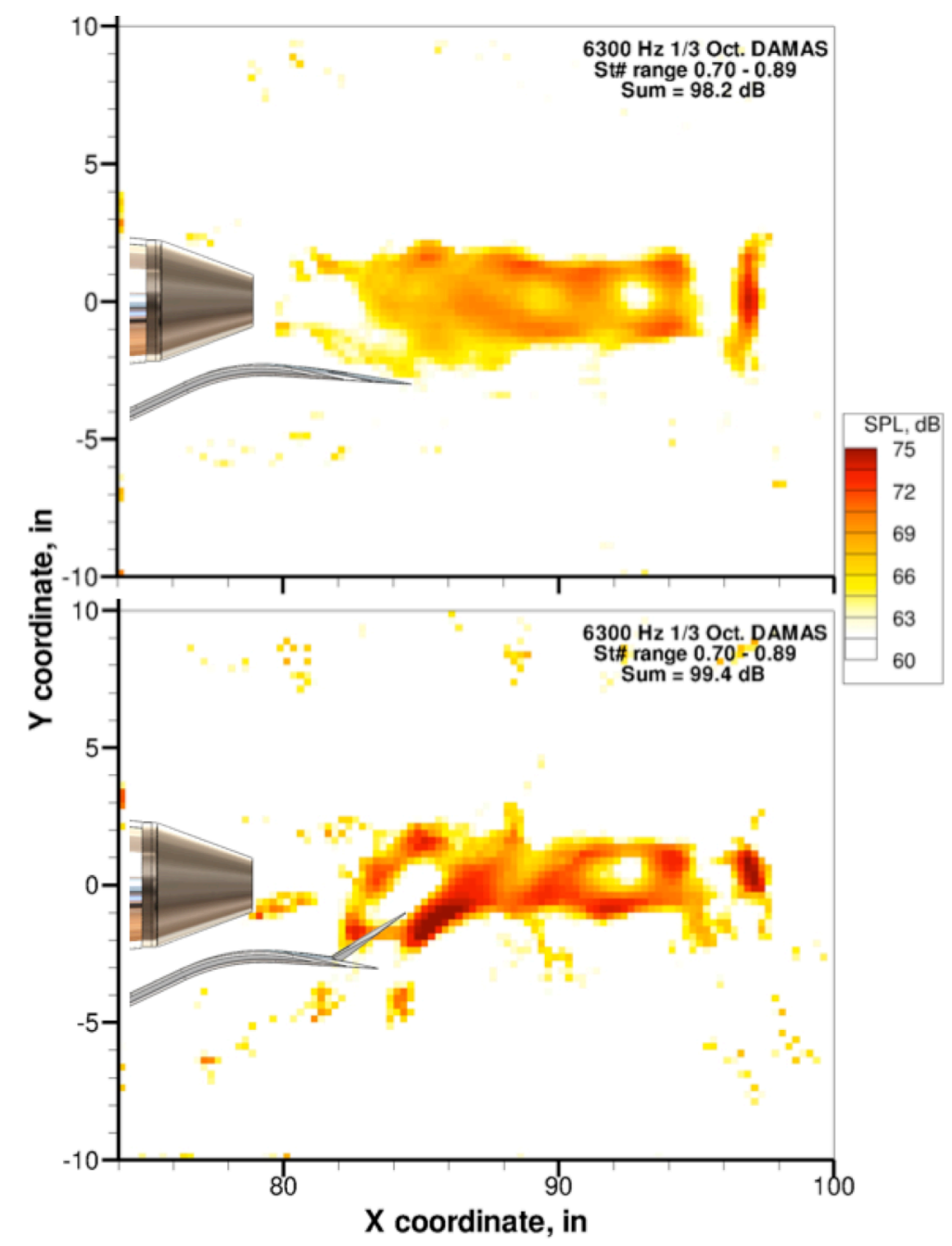

Figure 18. DAMAS results in the in-plane configuration comparing the $S / D=3.23$ spacing with $0^{\circ}$ elevon deflection (top) to the $40^{\circ}$ upward elevon deflection (bottom). DAMAS scan plane located along port jet centerline (closer to observer). Flow conditions are identical to those of Fig. 17.

\section{Shielding}

The twin jet model system with aft deck fuselage is rotated to the out-of-plane orientation to investigate shielding effects of this particular geometry. In Figure 19 JEDA single microphone spectra for the shielded configuration are compared to the unshielded case. In addition, a $5^{\circ}$ canted, shielded case, as well as the single microphone $+3 \mathrm{~dB}$ shielded case, are also shown. For $81^{\circ}$ to the jet inlet axis, Figure 19a exhibits the expected benefits of shielding at the higher frequencies in which the sources are closer to the nozzle exit. At lower frequencies there is no evidence of shielding and even some noise increases are seen with the shield in place. The overall levels show $1.2 \mathrm{~dB}$ OASPL benefit to shielding at $81^{\circ}$ and an additional $0.8 \mathrm{~dB}$ OASPL benefit with the canted jets. This indicates the noise benefit of inward cant angle previously shown in unshielded configurations is still effective in providing some additional benefit in a shielded configuration. At $129^{\circ}$ (Fig. 19b) the benefit of shielding is less noticeable, approximately $0.5 \mathrm{~dB}$ OASPL. It is also interesting to note that the shielded single jet $+3 \mathrm{~dB}$ case is similar to or lower than the twin jet shielded cases, suggesting the additional out-of-plane noise of twin jets observed in Fig. 13 is not completely shielded in this case. 


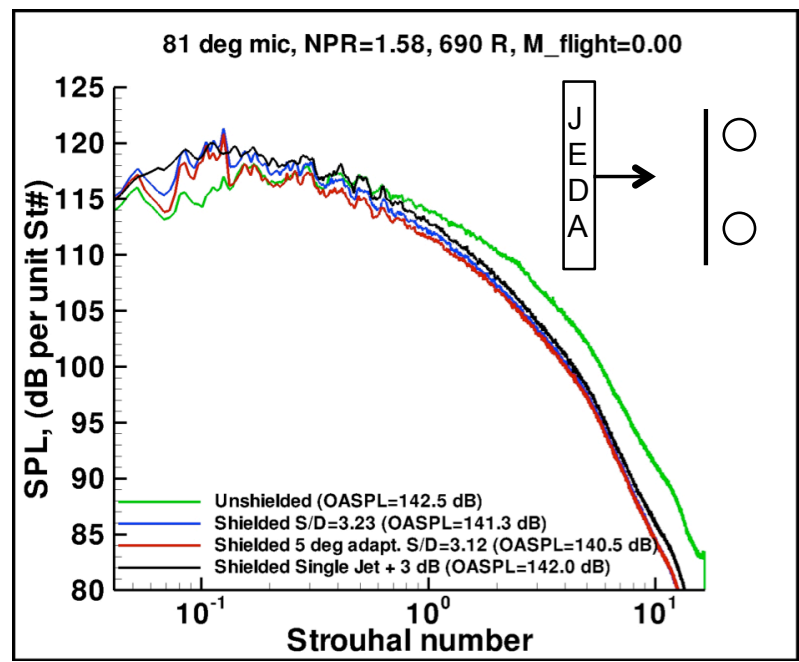

a)

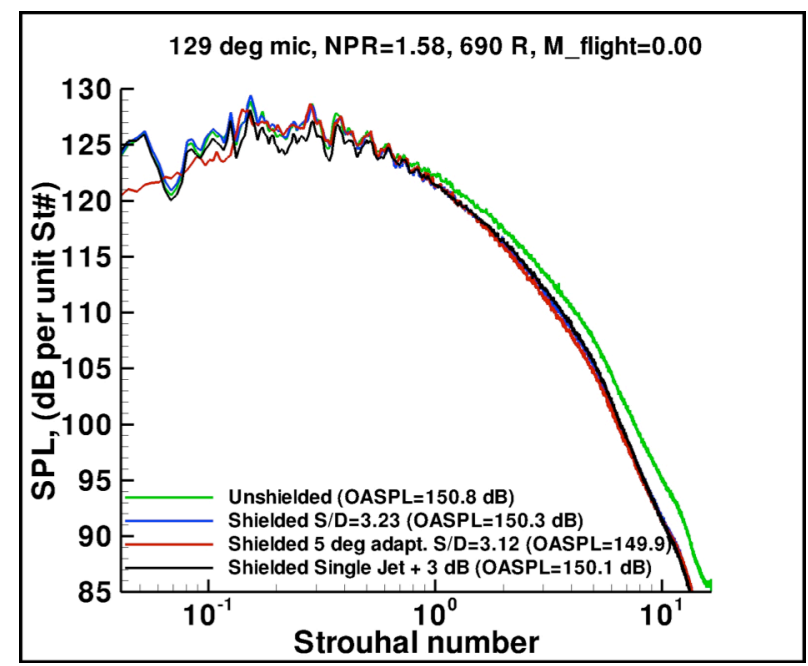

b)

Figure 19. JEDA microphone spectra showing the shielding effects for the out-of-plane configuration at a) $81^{\circ}$ and b) $129^{\circ}$.

Phased array results of this same out-of-plane configuration are compared in Figure 20. The aft deck shield is between the phased array and the jet nozzles, and the scan grid is a vertical plane running through both nozzle centerlines. The unshielded twin jet (top) is compared to both a shielded twin jet (middle) and a shielded single jet (bottom) in Fig. 20. As expected, the noise source maps for the shielded cases show no significant source strength upstream of the shield trailing edge. In fact, the source map does not show any noticeable source strength immediately downstream of the shield trailing edge either. This is likely due to some of the JEDA upstream microphones remaining in the shadow zone of the shield until further downstream of the trailing edge. An alternative approach is to apply the scan grid at the surface of the shield, the results of which are shown in Fig. 21. In this case the noise map does start at the shield trailing edge. The tradeoff is a less defined noise source map of the jet plumes.

To further address whether the DAMAS processing technique is effectively capturing the source field, each of the contour plots in Figs. 20 and 21 also contain the $6300 \mathrm{~Hz}$ third-octave level measured by a single microphone reference, in this case the JEDA center microphone. The integrated source map levels are within $0.5 \mathrm{~dB}$ or less when the scan plane is along the jet centerline. When the scan plane is along the shield plane, the integrated source map level is identical to the single microphone result, demonstrating the majority of the sound field is captured. Furthermore, as discussed in Brooks, et al. ${ }^{11}$ such results also suggest that much of the acoustic energy at this frequency can be modeled as incoherent. The tradeoff between good source resolution downstream and accurate capturing of the source at the shield trailing edge is under investigation. Possible approaches include having a multiplane scan plane or curved scan plane that captures both regions. 


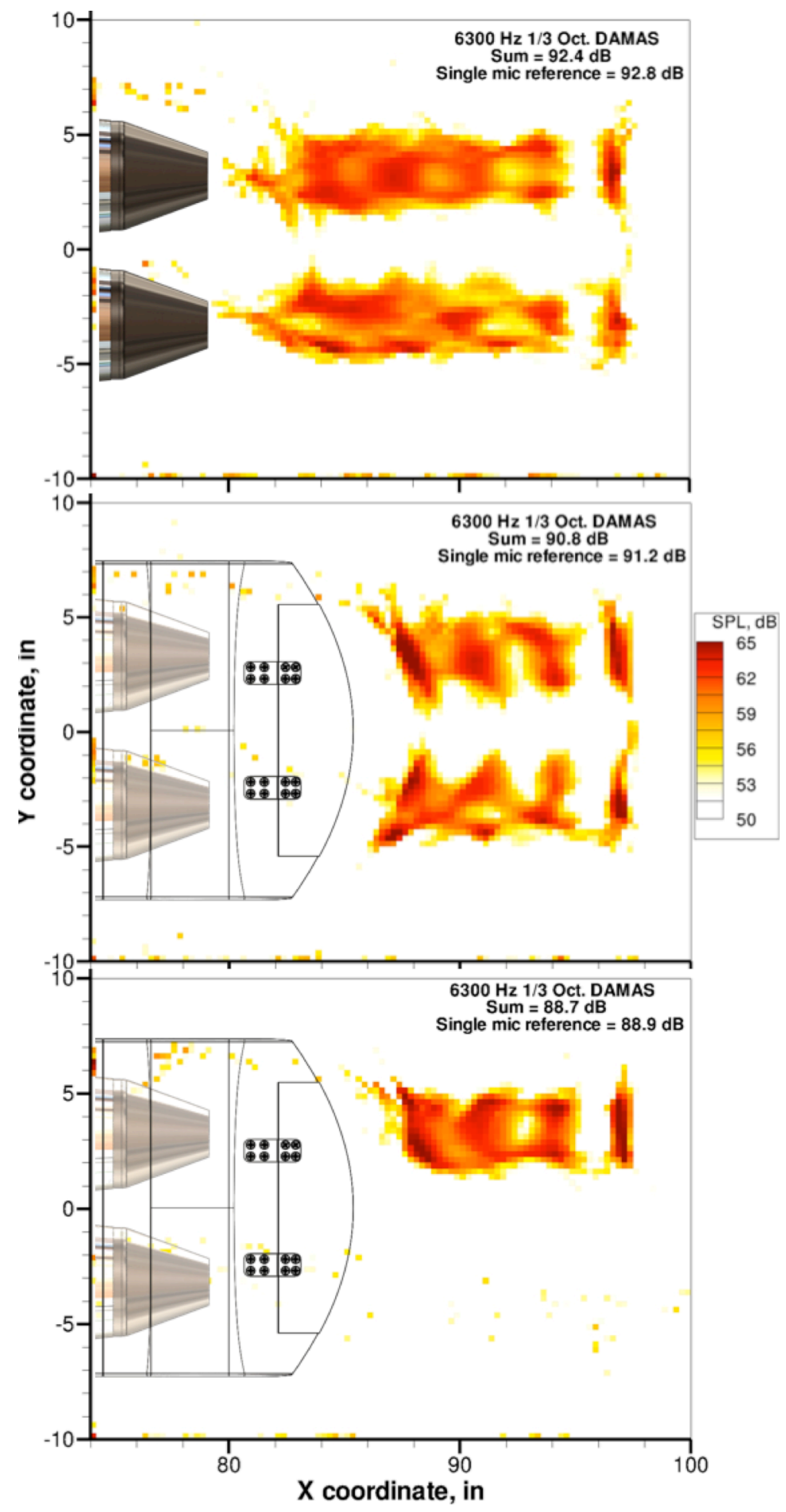

Figure 20. DAMAS results for $6300 \mathrm{~Hz}$ third-octave band in the out-of-plane configuration with spacing $\mathrm{S} / \mathrm{D}=3.23$. Comparison of unshielded (top), shielded (middle), and shielded with only top jet operating. Flow conditions are identical to those of Fig. 19, and the scan plane is through the jet centerlines. 


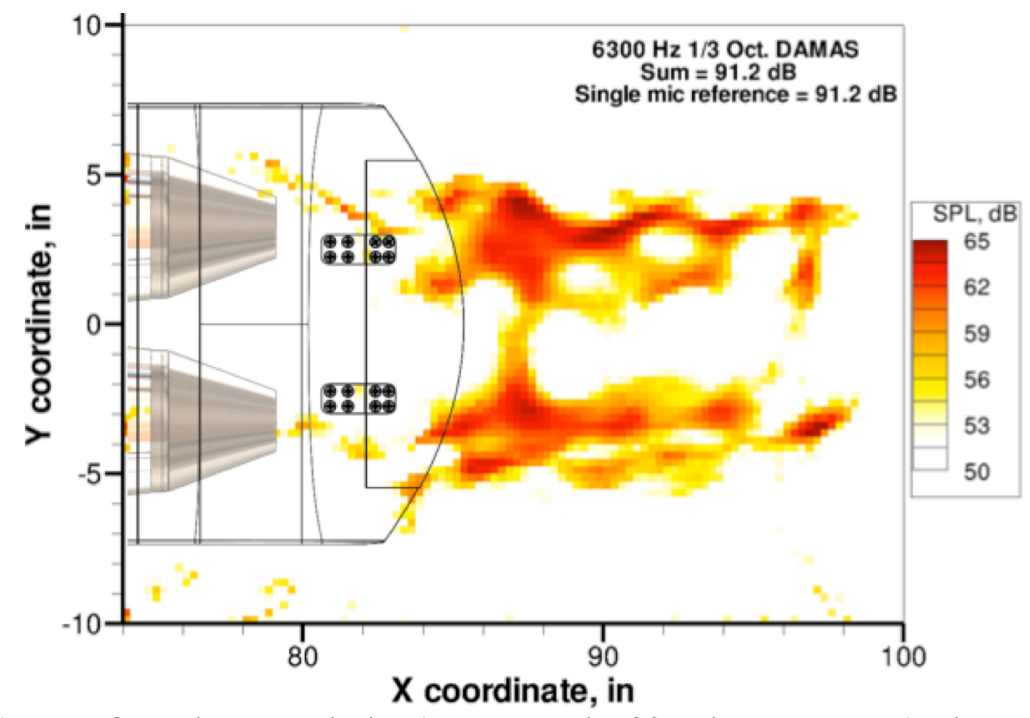

Figure 21. DAMAS result for shielded twin jet (compare Fig. 20, middle contour) with the scan plane now at the shielding surface instead of the jet centerline.

\section{Concluding Remarks}

This paper describes investigations to better understand the aeroacoustic properties of twin jets, particularly when in the presence of a hybrid wing body shield. This understanding is used to mitigate risks in an upcoming $5.8 \%$ scale Hybrid Wing Body acoustics test in NASA Langley Research Center's 14 by 22 foot wind tunnel. Here a twin jet nozzle system is operated in the Low Speed Aeroacoustic Wind Tunnel in conjunction with a $1.9 \%$ scale model of the aft section of the HWB fuselage. Mean flowfield, linear microphone array, and phased microphone array measurements are employed to focus investigations in four areas.

First, the acoustic effects of twin jet nozzle spacing are investigated in the out-of-plane azimuthal orientation using linear array microphones. Nozzle spacing has little effect at forward and sideline angles but impacts the acoustic spectra at further downstream angles as jets interact, peak levels increase, and spectral shapes are shown to change. The peak OASPL for each twin jet case investigated is greater than that of the single jet $+3 \mathrm{~dB}$ equivalent, and as the spacing between the twin jets increases, the peak OASPL moves to further aft angles.

Second, when canting two jets toward one another by $5^{\circ}$, the jet flowfields tend to merge at a location corresponding to the shear layer spreading angle plus the cant angle. In the out-of-plane configuration, acoustic results near $90^{\circ}$ to the jet axis indicate noise reductions of 1.3 to $2.1 \mathrm{~dB}$ OASPL for the $5^{\circ}$ canted jets at $\mathrm{S} / \mathrm{D}=2.27$, compared to the parallel twin jets. The out of-plane noise increases typical of twin jets seem to be mitigated by the more intense interaction of canted, closely-spaced jets. Phased array source maps also show a decrease in inner shear layer noise source strength for the canted, closely-spaced jets.

Third, in the in-plane configuration, the $40^{\circ}$ deflection of the modeled HWB center elevon is shown to increase noise by more than $5 \mathrm{~dB}$ OASPL at the upstream angles due to reflection, whereas noise at downstream angles is relatively unaffected or slightly shielded. Near $90^{\circ}$ to the jet axis the increase is approximately $1 \mathrm{~dB}$ across the entire spectrum. The phased array source maps show an increase in noise both at the elevon trailing edge and reflected in the upstream direction.

Fourth, the benefit of the aft deck fuselage shield is shown to be approximately $1.2 \mathrm{~dB}$ OASPL near $90^{\circ}$ and 0.5 $\mathrm{dB}$ OASPL near $135^{\circ}$ to the jet inlet axis. Additional benefits are demonstrated with the $5^{\circ}$ canted jets spaced at $\mathrm{S} / \mathrm{D}=3.12$. Furthermore, DAMAS source maps of shielded single jet and twin jet configurations with the scan plane at the jet centerline miss the source region at the shield trailing edge, likely due to shadow zone effects. Moving the scan plane forward to the shield surface effectively captures the source region downstream of the shield trailing edge but also results in less-resolved jet plumes. In addition, the integrated source map sound level for this case matches the single microphone measurement at the same frequency, suggesting the sound field is adequately captured. Work is ongoing to determine the optimal scan plane for these shielded applications. 


\section{Acknowledgments}

The author sincerely thanks the entire Jet Noise Lab team for their efforts during these experiments. In addition, Gerald Plassman was extremely helpful in supporting inquiries regarding DAMAS processing. Discussions with Tom Brooks and Tony Humphreys about DAMAS theory have also been very much appreciated. Funding from the Environmentally Responsible Aviation Project within NASA's Integrated Systems Research Program is gratefully acknowledged.

\section{References}

${ }^{1}$ Greatrex, F. B., and Brown, D. M., "Progress in Engine Noise Reduction," Proceedings of the First International Congress in the Aeronautical Sciences, Madrid, Vol. 1, 1958, pp. 364-392.

${ }^{2}$ Borchers, I. U., and Goethert, B. H., “An Experimental Study of the Noise Radiation of Interfering Free Jets," AIAA Paper No. 1977-1285, presented at the $4^{\text {th }}$ AIAA Aeroacoustics Conference, October 1977.

${ }^{3}$ Bhat, W. V., "Acoustic Characteristics of Two Parallel Flow Jets," AIAA Paper No. 1977-1290, presented at the $4^{\text {th }}$ AIAA Aeroacoustics Conference, October 1977.

${ }^{4}$ Parthasarathy, S. P., Cuffel, R. F., and Massier, P. F., “Twin-Jet Shielding,” Journal of Aircraft, Vol. 17, No. 9, 1980, pp. 618-626.

${ }^{5}$ Kantola, R. A., “Acoustic Properties of Heated Twin Jets,” Journal of Sound and Vibration, Vol. 79, No. 1, 1981, pp. 79106.

${ }^{6}$ Yu, J. C., and Fratello, D. J., "Measurement of Acoustic Shielding by a Turbulent Jet," AIAA Paper No. 81-2019 presented at the $7^{\text {th }}$ AIAA Aeroacoustics Conference, October 1981.

${ }^{7}$ Gerhold, C. H., "Two-Dimensional Analytical Model of Twin Jet Shielding," Journal of the Acoustical Society of America, Vol. 69, No. 4, April 1981, pp. 904-908.

${ }^{8}$ Simonich, J. C., Amiet, R. K., and Schlinker, R. H., “Jet Shielding of Jet Noise,” NASA Contractor Report 3966, 1986.

${ }^{9}$ Bozak, R., and Henderson, B., “Aeroacoustic Experiments with Twin Jets," AIAA Paper No. 2011-2790 presented at the $17^{\text {th }}$ AIAA/CEAS Aeroacoustics Conference, June 2011.

${ }^{10}$ Brooks, T. F., and Humphreys, W. M., “A Deconvolution Approach for the Mapping of Acoustic Sources (DAMAS) Determined from Phased Microphone Arrays," Journal of Sound and Vibration, Vol. 294, 2006, pp. 856-879.

${ }^{11}$ Brooks, T. F., Humphreys, W. M, and Plassman, G. E., "DAMAS Processing for a Phased Array Study in the NASA Langley Jet Noise Laboratory," AIAA Paper No. 2010-3780 presented at the $16^{\text {th }}$ AIAA/CEAS Aeroacoustics Conference, June 2010 .

${ }^{12}$ Amiet, R. K., "Refraction of Sound by a Shear Layer," Journal of Sound and Vibration, Vol. 58, No. 3, 1978, pp. 467-482. 